\title{
Recent Advancement in Biosensors Technology for Animal and Livestock
}

\section{Health Management}

\section{Suresh Neethirajan $^{1}$, Sheng-Tung Huang ${ }^{2}$, Satish K.Tuteja ${ }^{1}$, David Kelton ${ }^{3}$}

${ }^{1}$ BioNano Laboratory, School of Engineering, University of Guelph, Guelph, ON, Canada N1G

2W1

$8{ }^{3}$ Department of Population Medicine, University of Guelph, Guelph, Ontario, Canada, N1G 2W1

$9 \quad$ *Direct Correspondence to: BioNano Laboratory, School of Engineering, University of Guelph, Guelph, Canada 


\section{ABSTRACT}

The term biosensors encompasses devices that have the potential to quantify physiological,

27 immunological and behavioural responses of livestock and multiple animal species. Novel

28 biosensing methodologies offer highly specialised monitoring devices for the specific

29 measurement of individual and multiple parameters covering an animal's physiology as well as

30 monitoring of an animal's environment. These devices are not only highly specific and sensitive

31 for the parameters being analysed, but they are also reliable and easy to use, and can accelerate

32 the monitoring process. Novel biosensors in livestock management provide significant benefits

33 and applications in disease detection and isolation, health monitoring and detection of

34 reproductive cycles, as well as monitoring physiological wellbeing of the animal via analysis of

35 the animal's environment. With the development of integrated systems and the Internet of

36 Things, the continuously monitoring devices are expected to become affordable. The data

37 generated from integrated livestock monitoring is anticipated to assist farmers and the agricultural industry to improve animal productivity in the future. The data is expected to reduce

39 the impact of the livestock industry on the environment, while at the same time driving the new wave towards the improvements of viable farming techniques. This review focusses on the

41 emerging technological advancements in monitoring of livestock health for detailed, precise

42 information on productivity, as well as physiology and well-being. Biosensors will contribute to

43 the $4^{\text {th }}$ revolution in agriculture by incorporating innovative technologies into cost-effective

44 diagnostic methods that can mitigate the potentially catastrophic effects of infectious outbreaks 45 in farmed animals 


\section{Keywords: Biosensing; Nanotechnology; Precision livestock farming; disease diagnostics}

\section{Introduction}

Advances in engineering research and biomaterials, coupled with the decreasing costs of electronic technologies, have resulted in the emergence of 'sensing solutions' and smart computing technologies that include internet and cloud-based connectivity to develop integrated and networked physical devices for data collection and analysis. These systems are equipped to automatically collect data on physiological parameters, farm environment, production measures and behavioural traits.

In the modern world, new diseases that threaten animals' health emerge every year. There is currently a lack of reliable, cost-effective diagnostic tests for early detection of diseases in farmed livestock animals. Biosensing technologies have the potential to address these problems by developing innovative diagnostic tools for the rapid detection of key health threats within the agri-food livestock sector.

There are numerous factors that affect food production and have an influence on food security around the world. By 2050, food demand is expected to increase by $70 \%$, and meat production will increase by 50\%, making agri-food and livestock key industries for future growth (Alexandratos and Bruinsma, 2012). Health threats to animal populations can disrupt food supply chains and commerce with potentially long-lasting effects on human health, as well as economic impacts. With novel infectious agents and global pandemic factors on the rise in farmed livestock industries, efficient and timely strategies for monitoring and predicting risks are 
crucial. With current technology, detecting diseases in the early stage requires time-consuming

71 and expensive laboratory tests. There is a need for detection tools that can predict when an

72 incident is likely to occur and in what population, inform diagnosis and treatment options, and

73 forecast potential impacts on a given population (both human and animal). Furthermore, such

74 technologies must be accurate, affordable and broadly available. Strengthened laboratory and

75 field capabilities are needed to support these capacities. Diagnostic tools provide crucial

76 information to surveillance programs in diverse operational contexts, including networks and

77 reference diagnostic laboratories associated with the World Organization for Animal Health

78 (OIE), the United Nations Food and Agriculture Organization (FAO), and the Canadian Food

79 Inspection Agency (CFIA). These systems not only integrate the data on individuals and groups;

80 they can also help with the decision-making process by assisting in the early detection of health

81 issues and wellbeing problems in individual animals. These integrated systems will also help in

82 the implementation of corrective measures and improvements in management processes for

83 animal husbandry practices. The biosensor market for the year 2013 was valued at US \$11.39

84 Billion and is expected to increase to US\$22.68 Billion by 2020. This growth in the biosensor

85 market and associated applications is attributed to an increase in the demand for point-of-care

86 testing. Furthermore, non-invasive health monitoring is also driving the growth and development

87 of nanotechnology-based biosensors (Research, 2014). The precision farming market, important

88 in livestock management, is expected to grow from USD 3.20 Billion in 2015 to USD 7.87

89 Billion by 2022 (MarketsandMarkets). The driving socio-economic and environmental factors

90 that are expected to further the research and development to provide food for the growing human

91 population are detailed in Figure 1. 


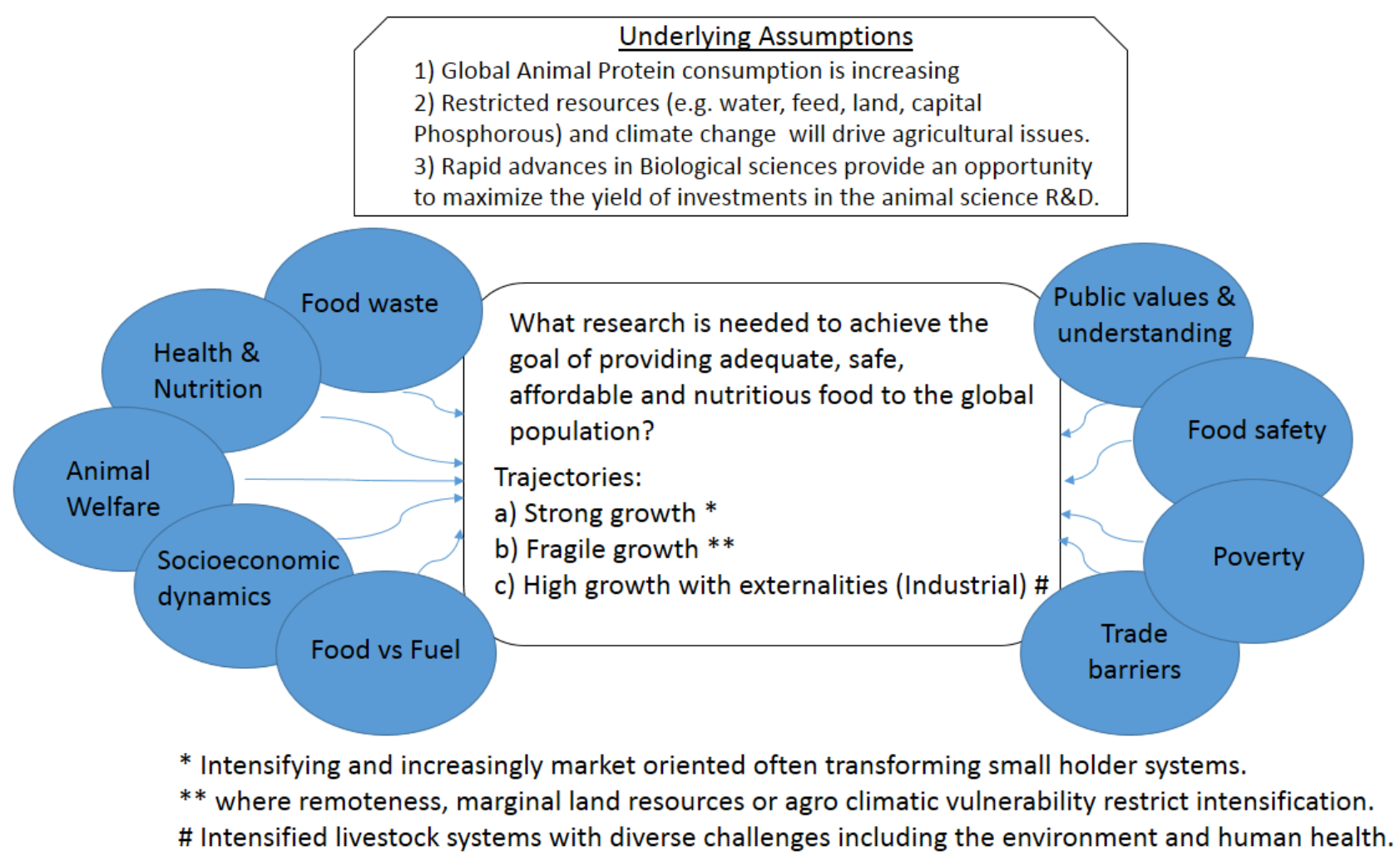

Figure 1. Schematic representation of environmental and socio-economic factors impacting 
We have entered a 'fourth revolution' in agriculture. This denotes the proliferation of new technologies including the Internet of Things, precision agriculture and mobile apps for disease surveillance. This review study will covers the technologies that will improve the capacity and efficiency of novel infection diagnosis, providing information needed to formulate sustainable risk assessment-based infection control programs for agri-food and livestock producers.

112 transform management in the livestock industry and the methods associated with it. One of the 113 salient features of biological, agricultural and environmental applications of nanotechnology is

114 that the nanoscale devices and systems are of the same size-scale as biomolecules. While 115 conventional sensors have been used in livestock monitoring, and as tools to assist in health 116 monitoring and disease diagnosis, nanobiosensors have the ability to multiplex the bioassays on117 site, thereby eliminating the need for the transportation of biological samples to centralised 118 laboratories for analysis. Integration of these sensors for wireless data transfer via a server or 119 through cloud-based systems would enable access to the analysed data for any internet-enabled 120 device. Nanobiosensor applications will not only reduce the incumbent costs for reagents, sample 121 handling, analysis times and transportation costs, but will also help in adapting and promoting 122 sustainable agricultural techniques and ethical handling of livestock.

124 These technologies shall focus on the non-invasive methodologies to assess animal welfare by 125 quantifying the stress and metabolic disease biomarkers, welfare assessment based on activity of 126 the animals (monitoring oestrus and lameness detection to maximise animal production) and 127 sensors for temperature and $\mathrm{pH}$ sensing (to determine calving alert and rumen function). 
Furthermore, various non-invasive sensing technologies for early disease detection shall help in saving animals' lives as well as reducing expenses for the farmers. A combination of these technologies and the use of 'smart' husbandry support systems will ensure maximum

131 productivity while improving the wellbeing of farm animals (Caja et al., 2016). Non-invasive 132 technologies for the chemical and biological analysis of samples from livestock, food and feed 133 can rapidly provide detailed information to evaluate the safety of various biological samples. 134 Biosensors equipped with robust data collection and integration infrastructure will be able to 135 realise this potential and shall become vital elements in real-time analysis of industrial agriculture. Not only will such systems help in maximising the utilisation of resources for

137 farming; they will also allow for an evaluation of individual and group behaviour of animals.

138 Development of on-site biosensing technologies will enable rapid, cost-effective and meaningful 139 monitoring of dietary inputs, environmental conditions, genetic makeup, performance, 140 metabolism, welfare and physiological state of animals.

\subsection{Biosensing - Taking a systems biology approach}

144 The application of biosensors in animal husbandry and agriculture will increase competitiveness

145 in the ever-changing global economy. The enormous amount of data generated by the continuous 146 monitoring shall generate new knowledge on animals' health and physiology and is expected to 147 result in the development of technologies that will improve efficiency of animal production, 148 better usage of dietary resources, improved health and welfare of animals through improved 149 animal management, and reduced output of waste per unit of food product, thereby decreasing the impact of animal production systems on the environment. 
151 Taking a systems biology approach to animal productivity and wellbeing is the way forward in

152 animal husbandry, as well as in agriculture. It will also be indicative of future human

153 performance and wellbeing initiatives. Collecting individual animal data as opposed to just 'herd

154 management' will be necessary to monitor the wellbeing of individual animals as well as animal

155 groups, and will help in identifying diseased animals sooner so as to provide healthcare and

156 prevent any disease outbreaks. Moving agriculture (animal productivity and wellbeing) on a

157 parallel course with human medicine and social science (human productivity and wellbeing) will

158 enable the determination of multi-parametric data on physiological and environmental factors

159 affecting animal welfare and productivity. This integrated database can be used to implement

160 best farm practices to ensure animal welfare and productivity and to predict animal behaviour.

161 Biosensing applications for livestock management and welfare will foster productive, value-

162 added partnerships in ways that will lead to social, health, environmental and economic benefits.

163 The approach to develop new solutions ranges from involving molecules to ecosystems, from

164 nanotechnology to big data analysis and management, and from microbes to sheep to human

165 populations. Moreover, real-time monitoring of animal health and assessment will have a direct

166 impact on animal productivity and better utilisation of resources. The future of biosensors lies in

167 utilising the comprehensive knowledge of animal physiology, genetics, environmental sciences

168 and animal nutrition, and integrating this knowledge in a meaningful way will aid in the

169 translation into real commercial and societal benefits. 
$173 \quad 1.2$ Integrated in "decision support systems"

175 Animals contribute to the human society in a number of different ways. These include being a

176 source of food, acting as models for studying human metabolism and diseases, and providing

177 companionship and services. However, considering that the human population is expected to

178 surpass 9 billion by 2050, feeding the population represents a formidable challenge. The global

179 demand for food is expected to increase by more than $50 \%$, while the consumption of animal

180 food and food products is expected to rise by $73 \%$ by the year 2050 (Alexandratos and

181 Bruinsma, 2012). The increase in human population demands increased availability of food using

182 sustainable methods and implementation of strategies to increase animal productivity and

183 agricultural production. It also demands the strengthening of legal systems to ensure sustainable

184 food chain management. In a society increasingly concerned with the welfare of animals,

185 methods to ensure ethical practices for animal rearing and animal wellbeing need to be 186 implemented.

188 As the global demand for high-quality animal protein increases, it will result in a competition for 189 limited food and feed sources from plants, further pressuring the agricultural industry to increase 190 productivity of food and feed crops per unit area of arable land. The environmental impacts of 191 livestock production (e.g., on soil, water, atmosphere and forest reserves) are key challenges to 192 bear in mind as we devise plans and policies to manage and further develop the production 193 systems that are environmentally sustainable, economically viable, ethically acceptable, and 194 provide wholesome and nutritious food for animals and humans on a global scale. These 195 challenges undoubtedly demand effective, rapid application of our cumulative knowledge, and 
196 innovative technologies in managing and caring for animals. Moreover, strong scientific bases

197 for environmental policies, assessment of food attributes, safety of animal foods, policies and

198 regulations governing animal management and welfare guidelines must form an essential

199 platform for devising future technologies. Integrating data and knowledge gained from decades

200 of research in environmental sciences, animal husbandry, agricultural practices and animal

201 nutrition and behaviour with the modern integrated electronic systems will play a pivotal role in

202 the optimisation and management of animal health and wellbeing (e.g., precision livestock

203 management) and to improve sustainability of the supply chain for feed and food production.

204

205

This integration will be handled by decision support systems, which, to be most effective, must

206 be robust under varying conditions; include technologies for rapid (automated) data collection

207 via wireless data transmission systems (Ruiz-Garcia et al., 2009) (i.e., animal and environmental

208 sensors); have substantial computing capacity for data analyses; have systems optimised to

209 inform decision making and be reasonably easy to operate. The next breakthrough will be for

210 these systems to use 'real-time biometry,' functioning in real time to monitor and control

211 genotype, environment, wellbeing, productivity and animal product quality.

213 Development of novel methods for the real-time assessment and management of animal

214 productivity and wellbeing is essential for investigating how the health-related parameters are

215 affected by diet, animal husbandry, environmental factors, and genotype by environmental

216 interactions. One of the key aspects of managing an animal's environment will be to optimise

217 feed ingredients and food quality (i.e., the most critical environmental factor), while maximising

218 the use of co-products. The application of these novel integrative technologies shall be of interest 
219 primarily to the small livestock (chickens, pigs, fish, sheep), as well as companion animals (cats

220 and dogs).

221

222 The precisely controlled small-scale experimental investigations on these species, focussed on

223 improved understanding of physiology, are likely to be scaled up to pilot-scale and eventually

224 result in future commercial applications. As for the livestock species, the quality of final product

225 like meat, milk, eggs, etc. will require careful consideration for quality, with the ultimate aim of

226 commercialisation of these integrated systems to be adapted on a larger world-wide scale where

227 data and inputs from animals are directly incorporated for use in the management software.

228

$229 \quad 1.3 \quad$ Biosensors will manifest themselves as indispensable tools in animal husbandry

230 1.3.1. Innovation and development for new approaches

231 Future developments in biosensors are expected to result in the development of new

232 methodological and technological approaches to measuring dynamic changes in real time, with

233 respect to the changes in physiological state and metabolism (e.g., gastrointestinal flora,

234 circulating levels of anabolic and catabolic hormones, immune function, gene expression). This

235 is to better understand the factors influencing animals' responses, and to develop solutions (e.g.,

236 husbandry practices, technology and associated decision support system) that improve

237 productivity and/or wellbeing of these animals.

238

239 1.3.2. Real-time data acquisition and analysis

240 Monitoring of real-time autonomic responses (e.g., respiration rate, heartrate and heartrate

241 variability, blood pressure, changes in peripheral blood flow) and defence-related reflexes (e.g., 
242 startle) using novel biosensing tools will help to investigate how housing, diet and genotype

243 affect animals' resiliency to stressors. These sensors will help in the understanding of factors that

244 influence the wellbeing of animals, and in the development of solutions (e.g., husbandry

245 practices, genotype selection) that improve the welfare of livestock and companion animals.

246 Advances in wearable or imprinted biosensors that are flexible and allow data transfer remotely

247 will be of special significance in this advancing area (Neethirajan, 2017) .

\subsubsection{Rapid characterisation of food and feed}

250 Biosensors shall be used to develop approaches enabling the rapid, accurate characterisation of

251 dietary inputs and final products (meat, eggs, milk) in terms of nutrient content (total and

252 bioavailable), anti-nutritional factors and bioactive components, as well as chemical and

253 microbiological contaminants, with the aim of implementing this technology at the level of the

254 commercial feed mill or animal food product processing plant. On the other hand, they would

255 also help in the decision-making process to alter the composition of feed to the animals in case

256 the animal products deviate from the expected nutritional status.

\subsubsection{Animal trait analysis and selection of robust breeds}

259 Biosensing may also help to select special animal breeds that are robust and resilient to

260 environmental stressors by enabling rapid assessment of the impacts of animal genotype and

261 environmental factors at different life stages. Such assessment would yield critical knowledge to

262 better understand genotype by environment interactions, in order to improve production

263 efficiency and animal wellbeing. The developments in biosensing will also help us better predict

264 and manage the impacts of climate change on animal agriculture over the next several decades. 


\subsubsection{Enabling planning of energy budgets and reduction of environmental impact}

266 The data collected and analysed using biosensors can assist in constructing detailed nutrient,

267 energy and elemental budgets for diverse livestock species at different life stages in response to

268 modulation in diet composition and environmental conditions, allowing precise management and

269 efficient usage of nutrients and minimisation of waste outputs. This will have a direct impact on

270 the efficient management of feed inputs and water resources while reducing the cost of

271 production, wastages and environmental footprint. For example, real-time monitoring of cattle

272 movement can provide information on the quality and quantity of forage, and the ability to

273 determine required changes to the grazing systems. Monitoring variables like the consumption of

274 water can provide insights into feeding behaviour, as well as the interaction between grazing

275 systems management and this behaviour. Quantifying animal water consumption within a

276 grazing environment can help to identify the impact of animal grazing on water quality, as well

277 as land utilisation. (Davis, 2007a; Davis, 2007b)

278

\subsubsection{Development of mathematical algorithms for better understanding of complex biological}

280 systems and their interaction with the environment

281 In the present day and age of big data, the data from animal farms is expected to help in the

282 development of advanced bio-mathematical models that are able to integrate data from the

283 aforementioned scientific research efforts and theoretical understanding of complex biological

284 systems. These models and simulations will allow for an improved quantitative appreciation of

285 the scientific and management aspects of animal agriculture. These will enable the assessment of

286 changes in the system with respect to different production, genetic selection, nutritional and

287 environmental factors. Ultimately, these models will help to identify approaches and strategies to 
288 improve the productivity, efficiency and wellbeing of animals and mitigate the potential negative

289 environmental impacts of livestock production. These models will also provide the basis for the

290 development of the specific algorithms required by a variety of decision support systems.

291

292 Current research and development in the field of biosensors for animal health management

293 focusses on innovative non-invasive, wearable sensors equipped with electronic systems for data

294 collection and transmission of data wirelessly. For specific areas where wearable sensors cannot

295 be multiplexed, especially in disease diagnosis, the development of portable hand-held systems

296 with immediate readout of results will enable fast decision-making processes and help in rapid

297 management of animal diseases. For example, non-invasive screening has been applied to the

298 detection of foot-and-mouth disease using hand-held air samplers with electrostatic particle

299 capture. In this case, infectious viruses are captured and subjected to analysis by real-time PCR

300 (polymerase chain reaction). Such biosensors can hasten the process of monitoring, diagnosis

301 and isolation of contaminated livestock in epidemiological contingencies (Christensen et al.,

302 2011; Wilson, 2015).

303

304 On the one hand, in the field, integrated biosensors will enable intensive (frequent and rapid)

305 evaluation of all aspects affecting an animal's behaviour, genetics and physiology, as well as

306 dynamic changes in metabolism and welfare. On the other hand, they will help manage the

307 production and analysis of the animal's food composition and help in rapid screening of diseases,

308 which at present cost billions of dollars annually to the livestock industry worldwide. For example, application of nanobiosensors for rapid detection of foot-and-mouth disease in swine

310 has a potential to save costs as well as prevent the spread of infection to the uninfected animals. 
311 Advancements in biosensing technologies is also expected to focus on the use of non-destructive

312 chemical analysis technologies, such as near infrared spectroscopy (NIRS), nuclear magnetic

313 resonance spectroscopy (NMRS) and tunable diode laser absorption spectrometry (TDLAS) for

314 the rapid and detailed evaluation of a biological sample's chemical composition and safety.

316 All these exciting technologies need to be developed for seamless performance and validation,

317 before they can be used routinely in research and, ultimately, in a commercial setting. Use of

318 surgically modified animals (e.g., multiple intestinal cannulations, arterials/venous catheters,

319 heart rate telemetry devices) for serial sampling to correlate physiological and metabolic

320 indicators with other variables (e.g., the non-invasive monitoring of behaviour and stress

321 responses, e.g., thermal imaging to assess changes in blood flow). This intensive collection of

322 data from various sensors and complementary analyses will generate a vast magnitude of

323 information, which will need to be effectively collected, compiled, synthesised, securely stored

324 and analysed using a series of advanced statistical, bioinformatics and mathematical modelling

325 approaches. This will require the implementation of a well-integrated and robust data collection,

326 storage and computing infrastructure.

328 2. Monitoring jaw movement of cattle to know the grazing efficiency

329 Cattle grazing behaviour requires individual monitoring of cattle based on three important

330 parameters, including the location of the animal, analysing animal posture and the movement of

331 the animal, especially movements such as walking and movement of the jaw (Herinaina et al.,

332 2016; Nadin et al., 2012). Jaw movements define the grazing behaviour of the cattle, and there 
333 are three different classes of biosensors that can be used to identify such movements. These

334 include:

2.1. Mechanical sensors (pressure sensors), acoustic sensors (microphone) and

336 electromyography sensors

Early systems developed to quantify the feeding behaviour of cattle were created exclusively for research purposes. The IGER Behaviour Recorder (Institute of Grassland and Environmental

to the halter and the change in the tube pressure. The installed software can distinguish between

347 bites and chews (Rutter, 2000). Peaks are considered to be bites when they are a combination of

348 a major long peak followed by a smaller sub-peak, or a non-symmetrical peak in the absence of 349 the sub-peak (Nadin et al., 2012). The IGER Behaviour Recorder can also be used to estimate the feed intake with reasonable accuracy, using data on the number of chews and eating duration

351 (using correlation coefficients) (Pahl et al., 2016). Miscalculations in the pressure sensing arise 352 from practical considerations, i.e., due to variations in the tightening of the halter on individual 353 animals, which can result in different pressure values. Another practical issue relates to the measurement of the output wave signal, which can be altered if the halter is mounted too tightly 
noseband pressure sensor, consisting of a data logger, incorporated in the noseband to record the

357 jaw movements via a pressure sensor (Braun et al., 2013).

359 2.2. Monitoring jaw movement through acoustic sensing

360 Acoustic analysis of grazing behaviour has been shown to accurately identify chewing and

361 biting, and therefore can be used to estimate the food intake of cattle (Laca and WallisDeVries,

362 2000). Acoustic analysis allows differentiation of three types of jaw movements: chew, bite and

363 chew-bite, and microphones can be used to record the jaw sounds of a grazing animal (Ungar

364 and Rutter, 2006). The data can be used to classify ruminating behaviour (Benvenutti et al.,

365 2016; Navon et al., 2013) and are especially helpful in monitoring animal wellbeing.

367 Acoustic sensing of jaw movements can be classified based on the microphone location and

368 acoustic system classification. Detailed analysis of the systems currently in use are elaborated in

369 Figure 2. Some systems are simple and detect jaw movements based on 10-minute recordings of

370 grazing sessions on a camera, with an accuracy of 94\% (Herinaina et al., 2016; Navon et al.,

371 2013). Signal patterns are analysed by the machine-learning algorithms to determine intervals

372 between jaw movements, intensity of each jaw movement (observed as a peak in the time

373 domain), their duration, and their integration in a sequence of behaviours (Navon et al., 2013).

374 The discrimination is based on the signal patterns produced during biting and chewing in a 1-

$375 \mathrm{kHz}$ sound window: peak frequency, peak intensity, average intensity and their duration (Laca et

376 al., 2000). Clapham and colleagues have demonstrated a fully automatic Chew-Bite Real-Time

377 Algorithm for detection and classification of ingestive events during cattle grazing. The system

378 consists of a directional wide-frequency microphone facing inwards on the forehead of the 
bioRxiv preprint doi: https://doi org/101101/128504; this version posted April 19,2017 . The copyright holder for this preprint (which was not certified by peer review) is the author/funder, who has granted bioRxiv a license to display the preprint in perpetuity. It is made available under aCC-BY-NC-ND 4.0 International license.

379 animal, and coupled with the signal analysis and decision logic algorithm, it can detect and

380 analyse bites and chews with an accuracy of 94\% (Clapham et al., 2011). Milone et al. have

381 reported the detection and classification of bites, chews and chew-bites with the help of the

382 Hidden Markov model, which estimates the sequences of bites, chews and chew-bites using

383 acoustic spectrum characteristics like decibels, by each sound. The successful classification is

384 reported to range between $61 \%$ and $99 \%$ (Milone et al., 2012).

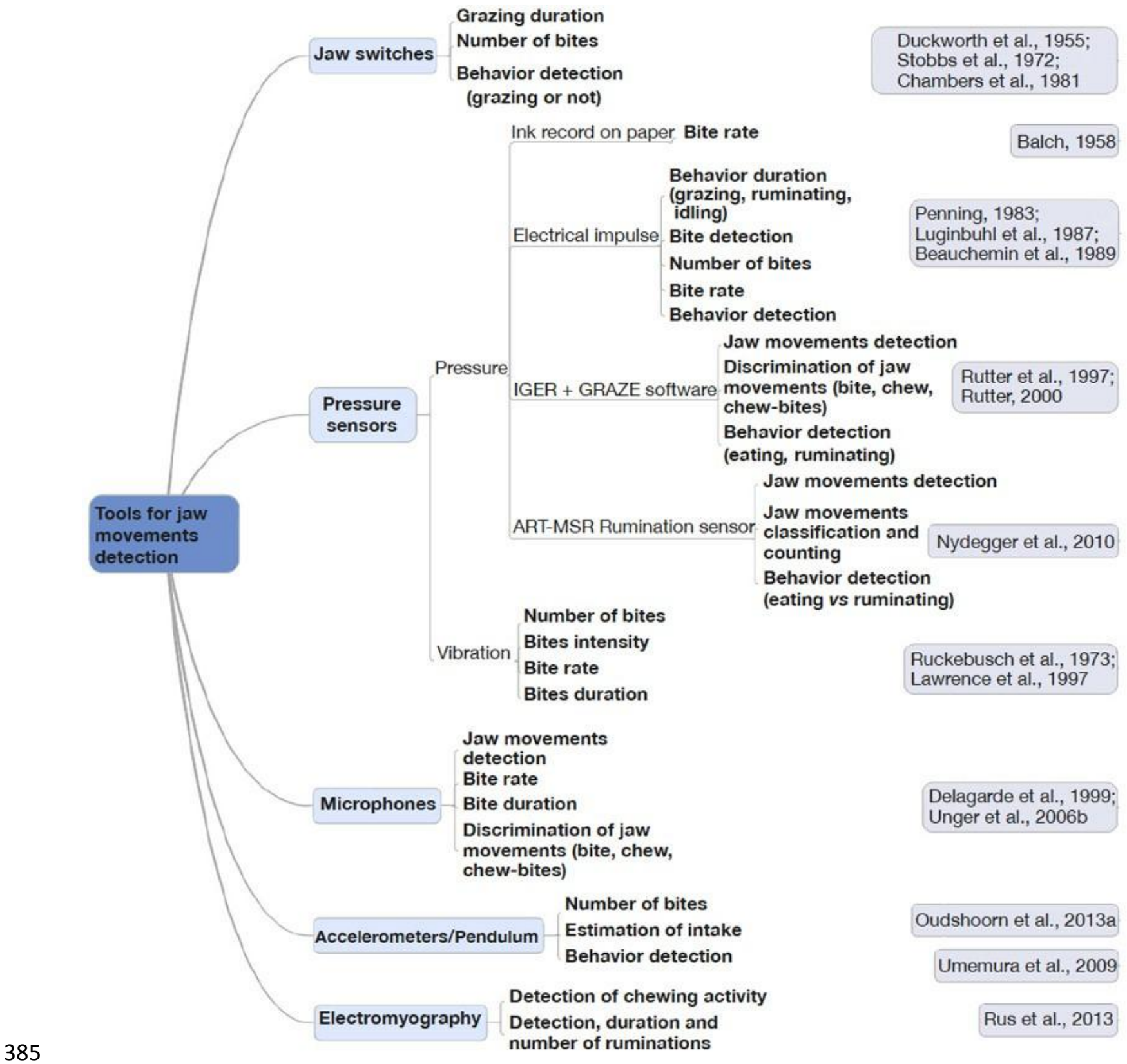


Figure 2. Primary tools used to detect jaw movements in cattle. Source: (Herinaina et al., 2016)

389 Microphone-based methods have demonstrated good accuracy for the detection of jaw movements and can differentiate between three different kinds of jaw movements. However, one

391 of the key disadvantages of acoustic systems in outdoor environments is their susceptibility to environmental noises. Acoustic interpretation techniques that can overcome these disturbances still need to be advanced before these systems are deployed on the farm.

\subsection{Acceleration sensors for jaw movement and feeding behaviour}

396 Accelerometer sensors convert physical acceleration recorded from motion or gravity into a 397 voltage output. Accelerometers can be used to measure static acceleration due to gravity, the 398 low-frequency component of the acceleration and the dynamic acceleration due to animal 399 movement (Herinaina et al., 2016). Several researchers have demonstrated the use of 400 accelerometers for analysing the grazing behaviour of animals (Mattachini et al., 2016; Tani et 401 al., 2013; Giovanetti et al., 2017). Andriamandroso et al. (Andriamandroso et al., 2015) used 402 smartphone inertial measurement units (IMUs) to count the number of bites through a frequency 403 pattern of single-axis acceleration data.

405 Oudshoorn et al. (Oudshoorn et al., 2013) were the first to use a 3-axis accelerometer to quantify 406 cow bites. The method involved visualisation of the recorded signals from the three individual 407 orthogonal axes to determine the signal that best matched the recorded bites. The method, 408 however, indicated an average correlation coefficient of 0.65 . Umemura et al. were able to 
monitor the jaw movements by modifying a pedometer into a pendulum, attached to the lower jaw. The data from the device could be downloaded wirelessly and the system showed $90 \%$

411 accuracy in measuring jaw movements when compared to manual counts over 10-minute

412 segments (Umemura et al., 2009).

414 One key issue associated with the use of accelerometers is the sensitivity of the signals recorded.

415 Undesirable signals due to rapid head movement or the movement of ears can interfere with data

416 interpretation, and the system would require a pre-processing of the signal relative to the jaw

417 movements in order to be useful for precision livestock farming. Interestingly, the IMUs, which

418 combine several sensors such as the accelerometers, gyroscope, magnetometer and GPS, can

419 offer a real advantage in terms of multiple-parameter measurements related to animal feeding

420 behaviour and animal position (active or not). Figure 3 details the key components of multi-

421 parametric sensors deployed for precision livestock farming. These multi-parameter sensors

422 collect and integrate the data from individual sensors to provide a comprehensive health picture

423 of individual animals, as well as herd behaviour. Such integrated sensors will also enable

424 predictions on animal health events and disease. In the event of a disease outbreak, multi-

425 parameter sensors can assist in identification and isolation of affected livestock before the spread

426 of the outbreak and potentially prevent unnecessary culling of uninfected animals, as is the

427 current practice in animal husbandry.

428

429 


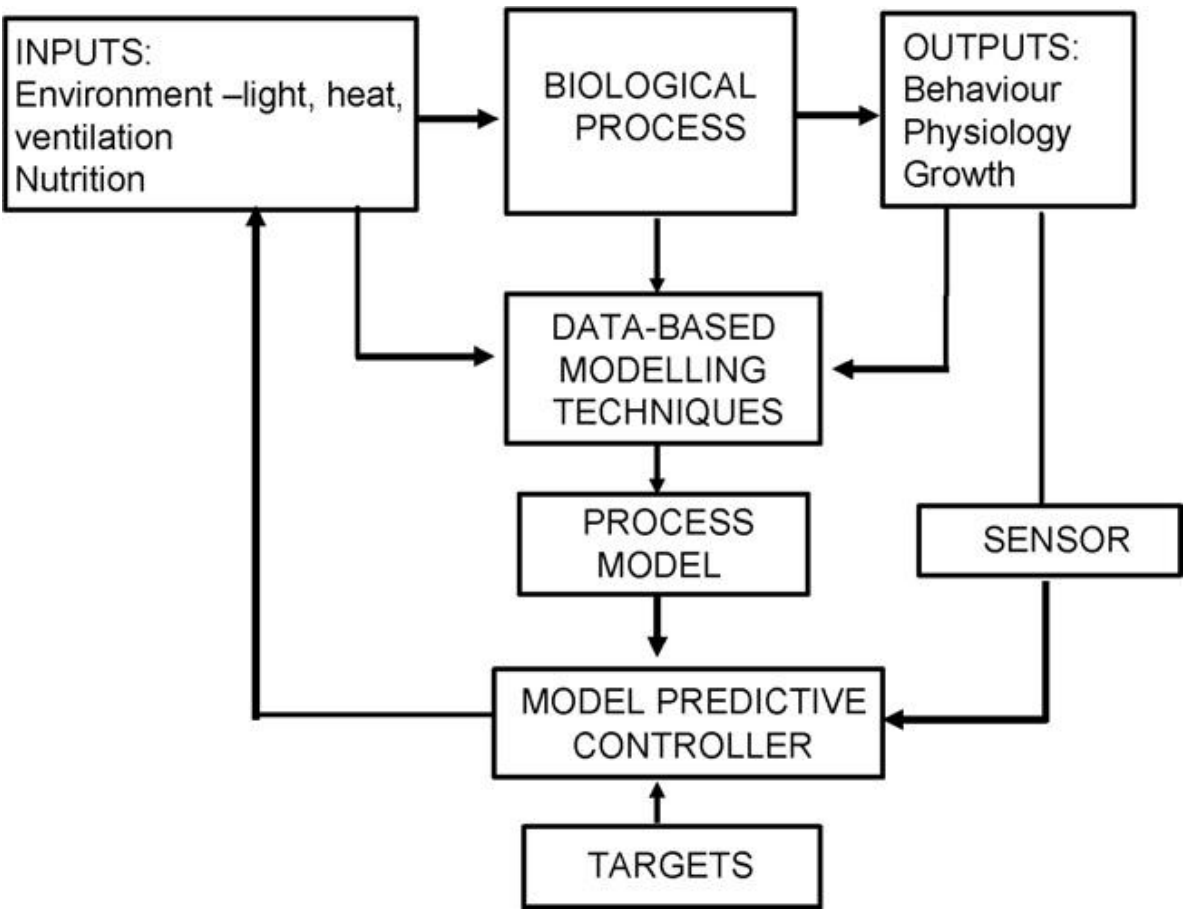

445

446 Figure 3. Schematic overview of the key components of Precision Livestock Farming to

447 control biological processes. Source: (Wathes et al., 2008) Reprinted with permission from 448 Elsevier Ltd.

\section{Biosensors for breath analysis}

451 Disease diagnosis by identification of volatile organic compounds (VOCs) has long been of 452 interest to researchers, as it offers a non-invasive methodology. VOCs can be found in the breath,

453 blood, faeces, skin, urine and vaginal fluids of animals as well as humans (Burciaga-Robles et 454 al., 2009; Garner et al., 2009; Spinhirne et al., 2004). These compounds are produced by a 
455 number of biochemical reactions, pathogens, and host pathogen interactions and are affected by a 456 number of biological variables such as age, actions, and biochemical pathways (Sethi et al., 457 2013). Breath monitoring provides a non-invasive and easy approach to determine the 458 physiological and general health status of animals. Advances in sampling methods, like solid459 phase and needle trap micro-extraction, and developments in techniques for representative breath 460 sampling (Turner et al., 2012) can be coupled with modern analytical technologies (spectroscopy 461 techniques and electronic noses) to allow for precise analysis of breath composition at an

462 unprecedented level (Pereira et al., 2015b). One of the key challenges that require important 463 attention is the statistical analysis and data interpretation of large and potentially heterogeneous 464 datasets collected from research on the exhaled breath composition from animals.

466 Metabolites in the breath include gasses like hydrogen and methane and volatile organic 467 compounds such as fatty acids, which can act as biomarkers for metabolic and pathologic 468 processes. Usually, the glucose level in blood is associated with VOCs like ketone bodies, 469 ethanol, methanol and exogenous compounds (Leopold et al., 2014).

471 In cattle, analysis of VOCs has been explored to diagnose bovine respiratory disease (Burciaga472 Robles et al., 2009), brucellosis (Knobloch et al., 2009), bovine tuberculosis (Fend et al., 2005; 473 Peled et al., 2012), Johne's disease (Kumanan et al., 2009), ketoacidosis (Mottram et al., 1999), 474 and normal rumen physiology. A rapid, non-invasive identification of foot-and-mouth disease 475 has been performed using air samples collected with a hand-held prototype device equipped with 476 electrostatic particle capture in a microchip chamber of 10-15 $\mu \mathrm{L}$ (Christensen et al., 2011). 


\section{$478 \quad 4 . \quad$ Sensors analysing metabolites in perspiration}

Most biosensors developed for analysing metabolites in sweat were developed with the purpose

481 of human health monitoring. These have been used to analyse sodium concentration (Schazmann

482 et al., 2010) and lactate levels, and converted to portable formats (belt form) to analyse sweat.

483 The electrochemical sensor for lactate levels includes a flexible printed tattoo that can detect

484 lactate levels with linearity up to $20 \mathrm{mM}$. The sensor has been shown to be resilient against

485 mechanical deformation. This sensor can also be adapted for use in animal sweat monitoring,

486 especially as a sign of physical stress in animals (Jia et al., 2013). Others have developed an

487 adhesive radio-frequency identification (RFID) sensor patch, which allows for potentiometric

488 sensing of solutes and surface temperature that can be read on a smartphone application (Rose et

489 al., 2015). New research in this area has led to the development of a fully integrated wearable

490 sensor array for multiplexed analysis of perspiration. The system includes a mechanically

491 flexible sensor array that measures metabolites like glucose and lactate, and electrolyte

492 composition such as sodium and potassium ions. The sensor also integrates temperature

493 measurement for comprehensive analysis (Gao et al., 2016).

495 Bovine tuberculosis (M. bovis) is a chronic bacterial disease affecting cattle and can occasionally

496 spread to humans by the inhalation of aerosols or consumption of unpasteurised milk. The ability

497 to identify volatile organic compounds produced by pathogens has been applied to this 498 technology for the detection of $M$. bovis infection by analysing the changes in the volatile 499 organic compound profiles present in breath. More recently, a proof of concept has been 
500 presented to reveal that the breath-derived volatile organic compound analysis can be used to

501 differentiate between healthy and M. bovis-infected cattle (Ellis et al., 2014).

502

503 The TB Breathalyser system for tuberculosis diagnosis in humans, developed by Rapid 504 Biosensor Systems, is already available in the market (McNerney et al., 2010). The gas505 chromatography/mass-spectrometry analysis has revealed the presence of VOCs associated with 506 M. bovis infection. A nanotechnology-based array of sensors has been tailored for detection of 507 M. bovis-infected cattle via breath, which allows real-time cattle monitoring (Peled et al., 2012).

508 Kumanan et al. have reported the development of a membrane-strip-based lateral-flow biosensor 509 combined with a high-throughput microtiter plate assay to enable highly sensitive reverse 510 transcriptase polymerase chain reaction (RT-PCR) -based detection of viable Mycobacterium 511 (M.) avium subsp. paratuberculosis cells in faecal samples (Kumanan et al., 2009).

\section{$513 \quad$ 5. Analysis of tears for continuous glucose monitoring}

515 Metabolites in tears can provide information about the concentration of these metabolites in 516 blood and provide a non-invasive continuous monitoring technique. Iguchi et al. have reported 517 the development of a flexible, wearable amperometric glucose sensor using immobilised glucose 518 oxidase on a flexible oxygen electrode (Pt working electrode and $\mathrm{Ag} / \mathrm{AgCl}$ counter/reference 519 electrode). The biosensor is fabricated using Soft-MEMS techniques onto a functional polymer 520 membrane (Iguchi et al., 2007). Others are working towards the development of a biosensor for 521 self-monitoring of tear glucose and are currently in the animal testing stages (La Belle et al., 522 2014) (Yonemori et al., 2009). 


\section{In vivo implanted biosensor to analyse stress in fish}

526 Fish health is affected by multiple environmental parameters as well as conditions in the fish

527 farms. Stressors include water pollution and changes in climate. Farm management practices like 528 stocking density and water exchange can also induce fish stress (Figure 4). Wu et al. have 529 devised an implantable biosensor that detects the composition of eyeball scleral interstitial fluid 530 in fish. The contents of the fluid correlate well with their concentrations in blood. Stress due to 531 changes in water chemistry, dissolved oxygen content, $\mathrm{pH}$, and metal toxicity were monitored, 532 and behavioural changes such as attacking behaviour and visual irritation were recorded (Wu et

533 al., 2015). Hibi et al. have developed a wireless biosensor system for continuous monitoring of 534 stress biomarker L-lactic acid in fish using the eyeball interstitial sclera fluid site for sensor 535 implantation. The biosensor allows for wireless monitoring of L-lactic acid in free-swimming 536 fish (Hibi et al., 2012). 


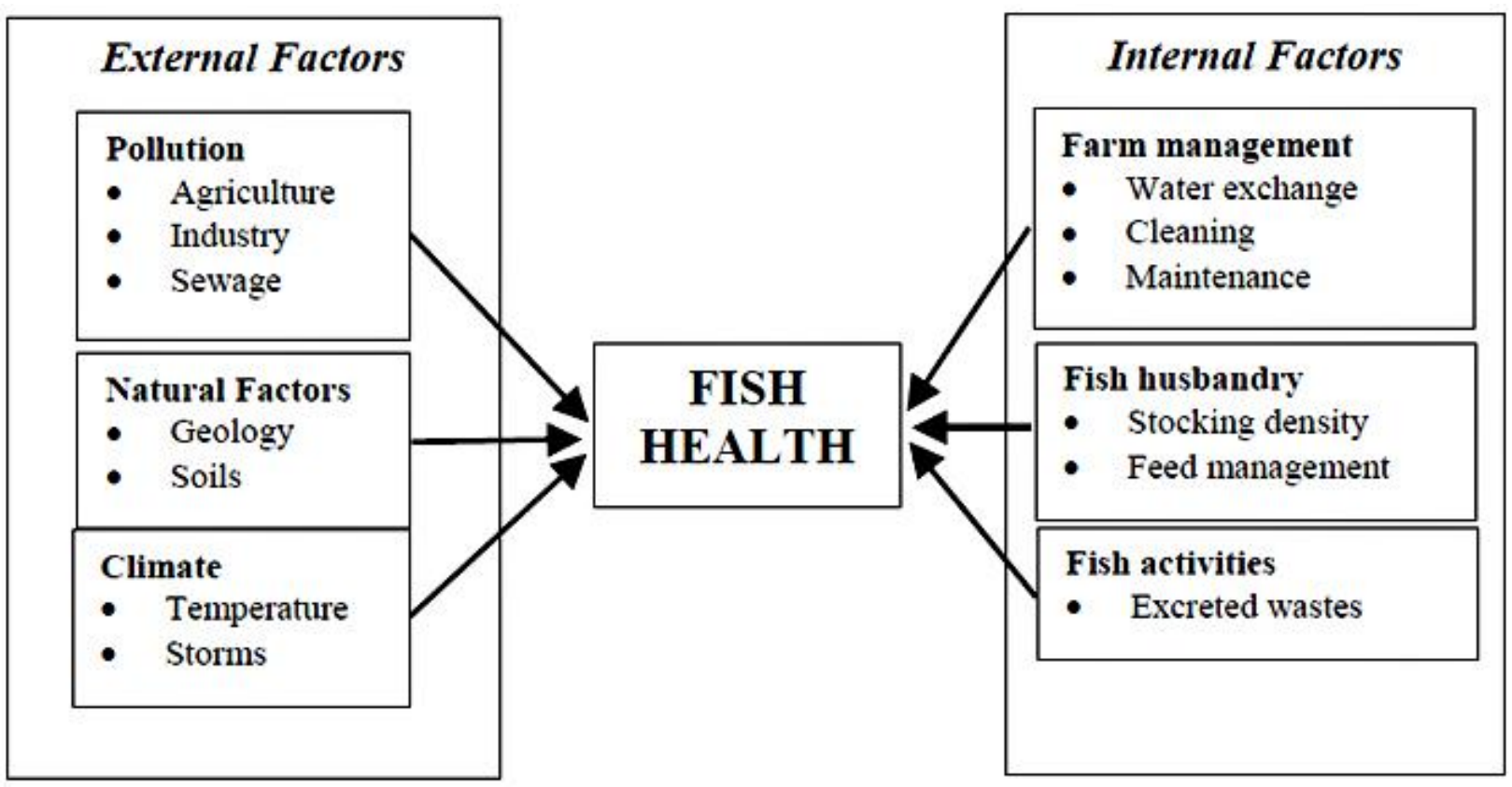

Figure 4. Environmental and farm management practices affecting fish health.

$543 \quad$ 7. Detection of ovulation

544 7.1. Progesterone

Breeding forms an integral part of livestock farming. Detection of the ovulation period in cattle

547 is important in order to determine the time window for artificial insemination. Conventional

548 oestrus detection involves ocular inspection of cattle by skilled labour, which is expensive as

549 well as inefficient. Biosensors for ovulation detection have been researched for a long time.

550 Pemberton and colleagues reported a device able to determine ovulation using a disposable

551 screen-printed amperometric progesterone biosensor, operated in a competitive immunoassay.

552 The biosensor included a monoclonal anti-progesterone antibody (mAb) immobilised on a 
553 screen-printed carbon electrode (SPCE). It was later incorporated into a thin-layer flow cell

554 offering advantages such as on-line analysis and improved fluid handling with the possibility of

555 future automation (Pemberton et al., 2001). The Herd Navigation ${ }^{\circledR}$ system was developed in

5562008 for commercial use and combines five sensing systems, including progesterone in the milk.

557 Herd Navigator ${ }^{\mathrm{TM}}$ (Durkin and DeLaval, 2010) measures the level of progesterone in milk and

558 the software suggests the insemination time, lists animals for final pregnancy confirmation,

559 indicates early abortion and lists the cows at risk for cysts and prolonged anoestrus (Mazeris,

560 2010). Oestrus detection rates of $95-97 \%$ have been reported in the farms in Denmark, with

561 significantly higher pregnancy rates (up to 42-50\%) than the conventional techniques (Blom and

562 Ridder, 2010; Vreeburg, 2010b). There are also reports indicating cost savings of $€ 250$ and

$563 € 350 /$ cow per year, as farmers do not have to spend money on expensive pregnancy tests

564 (Mazeris, 2010; Leonardi et al., 2013).

565

566 7.2. SPR-based biosensors for progesterone

568 More recently, Zeidan and colleagues have reported the development of a progesterone sensor by

569 integrating novel aptamer development with a nanoEnhanced Surface Plasmon Resonance

570 imaging sensor (SPRi). The authors first developed X-aptamers and selected them for binding to

571 progesterone. Then, the multi-array feature of SPRi was used to develop an optimised biosensor

572 capable of simultaneously screening the $9 \mathrm{X}$-aptamers for binding affinities. The sensor surface

573 was further optimised in a sandwich assay, where nanoEnhancers (NIR-streptavidin-coated

574 quantum dots) were used for ultrasensitive detection of progesterone molecules (Zeidan et al., 575 2016). 
576 7.3. Herd Navigator TM for monitoring ovulation

577 Herd Navigator has excellent oestrus detection rates, but the system is too expensive. Aiming at

578 improving detection reliability using low-cost sensor data, Jónsson et al. reported the

579 combination of information from step count and leg tilt sensors. The authors developed a

580 change-detection algorithm that can analyse cow-specific data in real time. The system has

581 shown an increase in the successful alerts and significantly reduced false positives (Jónsson et

582 al., 2011).

583

584 7.4. Intravaginal probes

585 Andersson et al. tested a wireless intravaginal probe, with a possibility for automation of the

586 process. The probe is based on the measurements of conductivity and temperature, and also

587 senses the movement of the animal. These parameters can all be used independently to detect

588 oestrus. Although still in the testing phase, the device has been shown to have a higher reliability

589 and to be more resistant to external disturbances as compared to existing alternatives (Andersson

590 et al., 2016; Andersson et al., 2015).

591

$592 \quad$ 8. Biosensors for animal diseases

593 8.1. Bovine Respiratory Disease

594 Bovine Herpes Virus-1 (BHV-1) is a major viral pathogen of Bovine Respiratory Disease

595 (BRD), the prominent cause of economic loss (\$2 billion annually in the US alone) to the cattle 596 and dairy industries. Tarasov et al. report the development of an extended-gate field-effect 597 transistor (FET) for direct potentiometric serological diagnosis of the BHV-1 viral protein via an 598 IgE-coated immunosensor. The biosensor was presented to be sensitive and selective to anti-IgE 
present in commercially available anti-BHV-1 antiserum and in real serum samples from cattle.

600

601

602

603

604

605

606

607

608

609

610

611

612

613

614

615

616

617

618

619

620

621

The system was shown to be faster than the traditionally used ELISA, amenable to multiplexing, and easily integrated into POC devices (Tarasov et al., 2016).

Schaefer and colleagues have investigated the use of an automated, RFID-driven, infrared thermography technology to determine BRD in cattle. The animals were monitored for BRD using biometric clinical scores, body temperature, haematology, serum cortisol and infrared thermal values. The data collected showed a correlation between animals positive for BRD with higher peak infrared thermal values of $35.7 \pm 0.35{ }^{\circ} \mathrm{C}$, in comparison to the true negative animals' $34.9 \pm 0.22^{\circ} \mathrm{C}$. The study is a proof of concept that the thermography data could be non-invasively and automatically collected on the basis of a system developed around the animals' water station (Schaefer et al., 2012).

\subsection{Detection of Bovine viral diarrhoea virus (BVDV)}

While ELISA and PCR-based methods have long been used for the detection of BVDV (Da Silva et al., 1995; Pritchard et al., 2002), rapid detection of BVDV requires an on-site monitoring and detection system to expedite the diagnosis and minimise the spread of disease in the herd. BVD disease affects beef and dairy industries worldwide, with severe implications to costs. Rapid diagnosis of BVDV through on-farm analysis is critical for herd protection and prevention of herd outbreaks. To this end, Montrose et al. have developed a fully integrated nanowire-based immunosensor to detect BVDV in serum. The biosensor has BVD virus as a capture molecule, which is covalently immobilised to a polymer electrodeposited onto a nanowire (Montrose et al. 2015). Luo et al. have developed an electrospun biosensor based on capillary separation and 
622 conductometric immunoassay for the detection of BVDV antibodies. The detection time of the

623 biosensor is 8 minutes, and the detection limit is $10^{3} \mathrm{CCID} / \mathrm{mL}$ for BVDV viral samples (Luo et

624 al., 2010). Heinze et al. have utilised microparticle immunoagglutination assays on a 625 microfluidic chip using forward light scattering measurements to detect BVDV particles (Heinze 626 et al., 2009).

627

628 8.3. Avian influenza virus

629 Avian Influenza Virus (AIV) infections have been a major cause of mortality, and rapid 630 detection methods for avian influenza have huge clinical, economical and epidemiological 631 implications. Diagnosis with ELISA and PCR is generally time-consuming and expensive, 632 requiring transport of samples to specialised laboratories. In recent years, there have been several 633 developments to miniaturise as well as provide assays that can be used on the farms for diagnosis 634 of disease. Diouani et al. and Wang et al. have reported the development of a miniaturised gold 635 electrode biosensor using impedance spectroscopy to detect $\mathrm{H}_{7} \mathrm{~N}_{1}$. The biosensor is based on the 636 detection of immobilised $\mathrm{H}_{7} \mathrm{~N}_{1}$ antibodies onto a bio-functionalised gold electrode (Diouani et 637 al., 2008) (Wang et al., 2009).

638

$639 \mathrm{Xu}$ et al. have developed an interferometric biosensor immunoassay for the direct and label-free 640 detection of avian influenza strains $\mathrm{H}_{7}$ (two strains) and $\mathrm{H}_{8}$ (one strain) through whole virus 641 capture on a planar optical waveguide. The assay relies on the index of refractive changes 642 occurring upon binding of virus particles to unique antigen-specific (hemagglutinin) antibodies 643 on the waveguide surface (Xu et al., 2007). Others have developed DNA-aptamers as recognition 644 elements in portable Surface Plasmon Resonance (SPR) -based biosensors for rapid detection of 
$645 \mathrm{H}_{5} \mathrm{~N}_{1}$ in swab samples from poultry (Bai et al., 2012). Luminescence resonance energy transfer

646 (LRET) -based biosensors for the ultrasensitive detection of the $\mathrm{H}_{7}$ strain (Ye et al., 2014) and

647 indium-tin-oxide thin-film transistors (ITO TFTs) on a glass substrate for immune detection of

$648 \mathrm{H}_{5} \mathrm{~N}_{1}$ antibodies have also been reported (Guo et al., 2013).

649

650 Others have developed quartz crystal microbalance (QCM) aptasensors based on ssDNA 651 crosslinked polymeric hydrogel for rapid, sensitive and specific detection of $\mathrm{H}_{5} \mathrm{~N}_{1}$ within 30

652 minutes (Wang and $\mathrm{Li}, 2013$ ) and QCM-based immunosensors to detect $\mathrm{H}_{5} \mathrm{~N}_{1}$ (Li et al., 2011).

653 Impedance-based sensitive and rapid methods for screening for the $\mathrm{H}_{5}$ subtype using immune-

654 magnetic nanoparticles have been reported, in which the virus is separated and the measurement

655 of an interdigitated microelectrode utilised for impedance measurement (Lum et al., 2012).

656

657 8.4. Foot-and-mouth disease

658 Rapid initial diagnosis of foot-and-mouth disease virus (FMDV) is essential for faster diagnosis.

659 Several biosensors have been developed recently to provide portable systems for the diagnosis of

660 FMDV. These have been reviewed extensively by Niedbalski (Niedbalski, 2016). The systems

661 developed include lateral flow immunochromatographic (LFI) for the detection of antibodies

662 against FMDV proteins (Yang et al., 2015; Yang et al., 2013) to detect FMDV serotypes O, A,

663 Asia 1, SAT 2 and non-serotype-specific FMDV. Several FMD-specific real-time RT-PCR (rRT-

664 PCR) assays have been made into portable mobile platforms for in-field detection of FDMV.

665 These include the Cepheid Smart Cycler Real-time PCR machine (Hearps et al., 2002), and the

666 BioSeeq-Vet (Smiths Detection). Genie I, a portable platform, also allows for the on-site

667 detection of viral RNA by reverse-transcription loop-mediated isothermal amplification (RT- 
LAMP) (Waters et al., 2014). Recently, rapid identification of FMDV has been reported using SpectroSens ${ }^{\mathrm{TM}}$ optical microchip sensors. Selective identification of FMDV is conducted in minutes and displayed as a yes/no readout using a hand-held device (Bhatta et al., 2012). Infrared thermography (IRT), a quantitative method for the assessment of body surface temperature, can be useful for the early detection of FMDV in the field. Temperature screening can be used to isolate potentially sick animals at an early stage and prevent the spread of disease. Microarrays, designed for laboratory diagnosis of FMD, offer greater screening capabilities for FMDV detection and can be regarded as an alternative to classical diagnostic methods. However, the apparatus needs to be miniaturised and made portable before it can be used directly in the field.

\subsection{Automated Detection of Mastitis}

Mastitis is associated with the inflammation of the udder in cattle due to an infection by Staphylococcus aureus. Mastitis detection in milk is based on two milk quality aspects: the somatic cell count (SCC) and the presence of visibly abnormal milk in the case of clinical mastitis. Efficient detection of mastitis is essential in order to manage the infected cattle and progression to clinical mastitis (Hogeveen et al., 2010). Neitzel and colleagues have developed an indirect on-line sensor system based on the automated California Mastitis Test (CMT) in milk (Neitzel et al., 2014). Duarte et al. have reported the development of an immune assay based on coupling with magnetic nanoparticles, which is analysed using a lab-on-a-chip magneto resistive cytometer, with microfluidic sample handling (Duarte et al., 2016). Others have reported the development of selective amperometric biosensors for infected milk detection based on the quantification of the catalase enzyme, which is immobilised on a thin-layer enzyme cell (Fütő et al., 2012). 


\subsection{Subclinical ketosis}

693 Nanobiosensors can significantly aid in the real-time detection of beta-hydroxy butyrate from

694 blood or milk to assessthe energy balance of the animals.. $\beta$-hydroxybutyrate $(\beta \mathrm{HBA})$ is an

695 indicator of subclinical ketosis, a common disease in dairy cows. Subclinical ketosis is one of the

696 metabolic diseases associated with negative energy balance during the transition period, as well

697 as decreased milk yields, impaired reproductive performance and higher risk of clinical ketosis,

698 resulting in economic losses (Ospina et al., 2010). Weng and colleagues have recently reported

699 the development of the on-chip detection of $\beta$ HBA using a miniaturised, cost-effective optical

700 sensor. The authors report that the analysis can be completed in 1 minute and has a detection

701 limit of $0.05 \mathrm{mM} \beta \mathrm{HBA}$ (Weng et al., 2015b). In another study, a biosensor using quantum dots

702 (QDs) modified with cofactor nicotinamide adenine dinucleotide (NAD+) has been used for

703 sensing $\beta$ HBA concentration in a cow's blood and milk sample. The detection is performed on a

704 custom-designed microfluidic platform combined with a low-cost, miniaturised optical sensor.

705 The sensing platform has a detection limit better than the previous method at $35 \mu \mathrm{M}$ (Weng et

706 al., 2015a) (Neethirajan et al., 2016).

707

708 8.7. Detection of porcine reproductive and respiratory syndrome (PRRS) virus

709 Infection with the porcine reproductive and respiratory syndrome virus (PRRSV) results in

710 PRRS in pigs, also known as the blue-ear pig disease, causing reproductive failure in breeding

711 stock accompanied by respiratory tract illness in piglets. The disease costs the United States

712 swine industry around \$644 million annually according to a 2011 study (Holtkamp et al., 2013),

713 and recent estimates in Europe found that it cost almost $1.5 \mathrm{~b} €$ in the year 2013. Several 
714 immunodetection-based biosensors have been reported for the detection of PRRS and are

715 detailed in Table 1.

716

717 Table 1. Techniques used for the detection of porcine reproductive and respiratory syndrome

718 virus

\begin{tabular}{|c|c|c|}
\hline Methodology & $\begin{array}{c}\text { Detection limit for } \\
\text { PRRS virus }\end{array}$ & Reference \\
\hline $\begin{array}{l}\text { Near-infrared electrochemi- } \\
\text { luminescence biosensor }\end{array}$ & $380 \mathrm{pg} / \mathrm{ml}$ & (Shao et al., 2017) \\
\hline $\begin{array}{l}\text { Platinum nanotube-based } \\
\text { fluorescent immuno-assay }\end{array}$ & $2.4 \mathrm{ng} / \mathrm{mL}$ & (Chen et al., 2015a) \\
\hline $\begin{array}{l}\text { enzyme-linked aptamer-antibody } \\
\text { sandwich (ELAAS) }\end{array}$ & $4.8 \mathrm{TCID} 50 / \mathrm{ml}$ & (Lee et al., 2013) \\
\hline $\begin{array}{l}\text { Immuno capture followed by } \\
\text { imaging epsillometry }\end{array}$ & $\begin{array}{l}2.4 \times 10^{3} \text { median cell } \\
\text { infectious dose }\left(\mathrm{CCID}_{50}\right. \\
\left.\mathrm{mL}^{-1}\right) \text { infectious virus }\end{array}$ & (Chen et al., 2013) \\
\hline Fluorescence Resonance Energy & & \\
\hline $\begin{array}{l}\text { Transfer (FRET) -based optical } \\
\text { biosensor using gold nanoparticles } \\
\text { and quantum dots }\end{array}$ & 3 viral particles/ $\mu 1$ & (Stringer et al., 2008) \\
\hline
\end{tabular}


Fluorescence resonance energy

transfer (FRET) -based using gold $\quad<25$ particles/ml (Grant et al., 2006)

nanoparticles

Piezoelectric quartz crystal-based

frequency changes

(Su et al., 2000)

720 8.8. Salivary detection of metabolites of clinical significance

721 Saliva sampling for disease and other biochemical markers of physiological health is an 722 attractive alternative to blood sampling, as it is non-invasive in nature (Bandodkar and Wang,

723 2014). The method is particularly useful for animal monitoring and disease diagnostics, as blood

724 collection from animals is considered to be a stress inducer and may have an impact on the

725 biochemical parameters being diagnosed. The ability to collect and immediately analyse the

726 salivary samples on-site provides numerous advantages for field applications. Biomarkers in

727 saliva can be helpful in numerous ways, e.g.: (i) early detection and diagnosis of diseases; (ii) in

728 supporting the decision-making processes for animal handling; and (iii) to monitor the

729 progression of disease (Malon et al., 2014). However, it must be noted that current analysis

730 procedures, if applied to saliva, would require huge amounts of salivary probes for the

731 biochemical assays. Although saliva sampling using oral fluid collectors and commercial devices

732 (Mottram et al., 2004) is generally safe and convenient to use and provides a sufficient

733 homogeneous sample with low viscosity, it still presents several shortcomings, such as (i) the

734 requirement of supervision; (ii) the need to follow the procedures carefully to ensure sample

735 adequacy; and (iii) it is a time-consuming process. Moreover, the assays for biomarkers in saliva 
736 have to be calibrated against the assays for blood samples to ensure the sensitivity of detection

737 and the robustness of the assays.

738 8.8.1. Biosensors for salivary uric acid

739 An abnormal concentration of uric acid acts as a biomarker for several diseases, such as

740 metabolic syndrome, renal syndrome, and abnormalities in purine metabolism (Nakagawa et al.,

741 2006; Nyhan, 1997). Uric acid is also known to be present in response to physical stress

742 (Hellsten et al., 1997). Detection of uric acid in saliva presents a non-invasive method, and there

743 is a good correlation between uric acid levels in blood and saliva (Soukup et al., 2012). Kim et

744 al. have reported the development of a wearable salivary uric acid mouth guard sensor in which a

745 uricase-modified screen-printed electrode system is integrated into a mouth guard platform. It

746 uses miniaturised instrumentation electronics featuring a potentiostat, a microcontroller, and a

747 Bluetooth Low Energy (BLE) transceiver. This platform enables real-time wireless transmission

748 of information to standard smartphones and other storage devices (Kim et al., 2015).

\subsubsection{Measurement of salivary cortisol as an animal stress biomarker}

751 Measurement of corticosteroid hormones is commonly used as a biomarker of an animal's

752 response to stress. The difficulties in obtaining blood samples and the recognition of the stressor

753 effect of blood sampling are primary drivers for the use of minimally invasive sample media.

754 Salivary cortisol has been established as a viable indicator of stress levels in animals held in

755 captive environments. The suitability of a cortisol assay and its validation have been detailed in

756 the review by Cook (Cook, 2012). More recently, Yamaguchi et al. have demonstrated the

757 development of a cortisol immunosensor for the non-invasive and quantitative analysis of

758 salivary cortisol. The immunosensor detects current resulting from a competitive reaction 
759 between the sample cortisol and a glucose oxidase (GOD)-labelled cortisol conjugate, and 760 quantifies cortisol levels based on a calibration curve. The technique takes 35 minutes for

761 analysing salivary cortisol levels and the device can be used on-site. The method was also shown

762 to closely correspond to the currently available ELISA method (Yamaguchi et al., 2013).

763

764

\subsubsection{Measurement of salivary glucose}

765 Salivary glucose biosensors have primarily been developed for human use and for domestic pets

766 (Reusch et al., 2006; Stein and Greco, 2002), but they have vast potential in monitoring livestock

767 health. Most of the sensors developed for humans have also been tested in animals. The sensors

768 are lightweight and portable and can be used directly on-field (Park et al., 2009).

770 8.9. In vivo real-time sensing for uric acid in poultry

771 Energy pathways in birds are lipid metabolism rather than the glucose metabolism dominant in

772 other animals, and uric acid levels are indicative of protein catabolism in birds (Gumus et al.,

773 2014). Numerous methods for uric acid detection based on chemiluminescence,

774 spectrophotometry, fluorescence (Martinez-Pérez et al., 2003) and electrochemistry (Jindal et al.,

775 2012) have been developed. However, Gumus et al. have reported the development of uric acid

776 sensing based on uricase enzyme for in vivo applications. It is an enzyme-based method using

$777 \mathrm{Pt} / \mathrm{Ir}$ wire and $\mathrm{Ag} / \mathrm{AgCl}$ paste. The sensor has a linear response for uric acid in the range of 0.05

778 to $0.6 \mathrm{mM}$ uric acid, which covers the physiological levels for avian species and is able to 779 transmit the data wirelessly. 
8.10. Salivary alpha amylase as a stress biomarker in pigs

782 While cortisol is an essential hormone responsible for the regulation of stress, salivary alphaamylase is a novel biomarker for psycho-social stress. This has been validated by Fuentes et al.

784 using an automated spectrophotometric method for salivary alpha-amylase measurement

785 (Fuentes et al., 2011). Wu et al. have fabricated an inexpensive, disposable $\alpha$-amylase biosensor 786 by immobilising a layer of starch gel on a thick-film magnetoelastic sensor. When exposed to $\alpha$ 787 amylase, the resonance frequency of the starch gel-immobilised sensor increases in proportion to 788 the starch hydrolysis by $\alpha$-amylase, allowing for its quantification. The sensor described can 789 detect 75 to $125 \mathrm{U} / \mathrm{ml} \alpha$-amylase (Wu et al., 2007).

\section{Livestock monitoring systems for observing physiological parameters and health of cattle}

792 Jegadeesan et al. have proposed a two-component system. The first component is the monitoring 793 and collection of data on the health parameters of animals in the field, and the second component 794 is the monitoring and acquisition of data on animals from the farms. Animals are subjected to a 795 variety of stress factors during their lives on farms. These include stressors due to changes in 796 temperature, transport across farms, physiological stress due to ill health or improper food intake 797 as well as stress due to restraint (detailed in Figure 5). This information on the external 798 environment and animal health can be collected and analysed in real time using closed-circuit 799 cameras. The complete system is expected to work independently, making necessary changes in 800 response to the real-time data inputs. Human intervention is only expected in the event of an 801 emergency (Jegadeesan and Venkatesan, 2016). Although such a model system is expected to be 802 a norm in the near future, steps are underway to implement these integrated systems at least 803 partly in livestock and agriculture. 
805 Contagious livestock diseases can result in economic losses and decreased productivity for cattle

806 farms. Park et al. have developed a livestock monitoring system (LMS) integrated with a

807 wireless sensor network to collect data on heart rate, breathing rate and cattle movement. The

808 authors report an average correlation coefficient of 0.97 for the collected data. Such systems

809 have the ability to change the landscape of animal farming by providing comprehensive

810 information on animal wellbeing. These systems are also able to rapidly identify livestock

811 diseases and prevent economic losses stemming from loss of productivity (Park and Ha, 2015).

Increased cortisol, catecholamines, and acute phase proteins in blood Increased heart rate

Altered leukocytes distribution Pro-inflammatory responses Increased vocalization, increased ambulation and aggressive behavior Increased respiratory disease
Altered feed intake and digestion Compromised reproduction Altered growth and body weight Decreased milk production Increased mortality Altered metabolic function

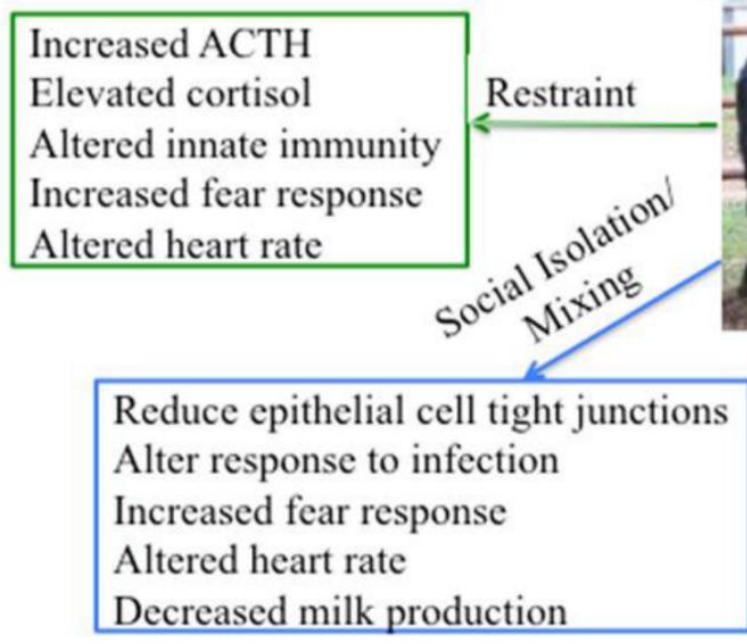

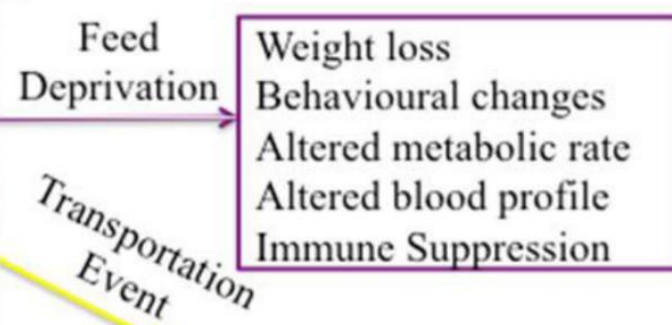

Increased mortality

Increased susceptibility to respiratory disease

Cortisolemia

Altered leukocytes distribution

Pro-inflammatory responses 
814 Figure 5. Impact of individual stressors on biological functions in cattle. Source: (Chen et al., 815 2015b)

817 9.1. Monitoring $\mathrm{CO}_{2}$ ventilation in farms

818 Carbon dioxide $\left(\mathrm{CO}_{2}\right)$ can be used as a tracer gas to measure ventilation, as well as emission 819 rates of $\mathrm{CO}_{2}$ (Persily, 2016). Currently, $\mathrm{CO}_{2}$ concentrations from agricultural facilities are 820 measured using a Photo Acoustic Spectroscope (PAS) gas analyser (Chepete et al., 2012;

821 Hassouna et al., 2013; Wheeler et al., 2006; Zhao et al., 2012) and the Open-Path laser (OP822 laser) (Frish, 2014; He et al., 2009). Although these are appropriate for use in mechanically 823 ventilated farms, the measurements in naturally ventilated farms show considerable variability. 824 Moreover, these systems are cost-intensive when multiple samples are required. Non-Dispersive 825 Infra-Red (NDIR) -based sensors are suggested as an alternative for PAS and OP-laser to 826 measure $\mathrm{CO}_{2}$ concentrations in $\mathrm{NV}$ buildings. Experimental evaluation of NDIR sensors to 827 measure $\mathrm{CO}_{2}$ levels has been demonstrated (Calvet et al., 2014; Piccot et al., 1994; Yasuda et al., 828 2012) along with the miniaturisation of the method, facilitating in-field usage (Hodgkinson et al., 829 2013). Mendes et al. have recently demonstrated the use of $\mathrm{NDIR} \mathrm{CO}_{2}$ sensors for monitoring $830 \mathrm{CO}_{2}$ levels in a naturally ventilated dairy cow barn, comparing them to other commercially 831 available NDIR $\mathrm{CO}_{2}$ sensors. The authors conclude that the $\mathrm{CO}_{2}$ concentrations were in 832 agreement with the platforms tested, and the number of NDIR sensors required to represent the 833 overall $\mathrm{CO}_{2}$ concentration of the dairy cow barn must be calculated based on the barn length and 834 occupied barn area. The NDIR $\mathrm{CO}_{2}$ sensors were found suitable to be used as a multi-point 835 monitoring system of $\mathrm{CO}_{2}$ concentrations in $\mathrm{NV}$ buildings as a feasible alternative to PAS and 836 the OP-laser methods (Mendes et al., 2015). 


\subsection{Monitoring animal movement and behaviour}

839 Monitoring movement and behaviour can provide information on an animal's activity and 840 wellbeing. A top-view camera can provide vital information if the animal is low-weight. Motion-

841 detection technology and video recording coupled with the Gaussian Mixture Model (GMM) can

842 be used to gather information on animal size and identify low-weight animals (Sa et al., 2015).

843 MooMonitor integrates information on cow oestrus, as well as data on rumination, feeding and

844 levels of activity. It makes use of wireless sensors for the two-way transmission of data. Other 845 technologies, such as HerdNavigator ${ }^{\mathrm{TM}}$ and the Afimilk Silent Herdsman also serve the same

846 purpose. The Silent Herdsman is a wearable technology and monitors all activities of cattle to

847 analyse their behaviour. Any changes in an animal's behaviour pattern can be used to identify the 848 oestrus cycles and onset of disease/sickness.

850 Honeybees produce a variety of different sounds as a means to communicate with the colony.

851 The sounds have characteristic low fundamental frequencies between 300 and $600 \mathrm{~Hz}$ (Barth et 852 al., 2005). Honeybees' sounds have specific frequencies within this range for a number of 853 reasons. Both the range of sound frequency and the acoustic signal pattern determine the 854 meaning of the sound. An accurate quantification of these signal patterns can give valuable 855 information on the hive health (Qandour et al., 2014). Electronic systems for management of 856 beehives have been developed lately and combine hive acoustics monitoring with measurement 857 of parameters like brood temperature (Kridi et al., 2016), humidity, hive weight and the weather 858 conditions of the apiary. Dietlein et al. have developed a system for automated continuous recording of sound emission by honeybees as a measure of their activity (Dietlein, 1985). The 
specific sounds from the hives are useful in providing information on colony behaviour, strength and health, and the data from the monitoring device can be accessed remotely from any internetenabled device (Bromenshenk et al., 2015; Evans, 2015; Meikle and Holst, 2015). Other systems are being developed that integrate visual, acoustic and beehive monitoring systems and share them with the environmental monitoring platform (Figure 6). These systems can help in collecting and analysing the data on bee behaviour for biologists (Chiron et al., 2013).

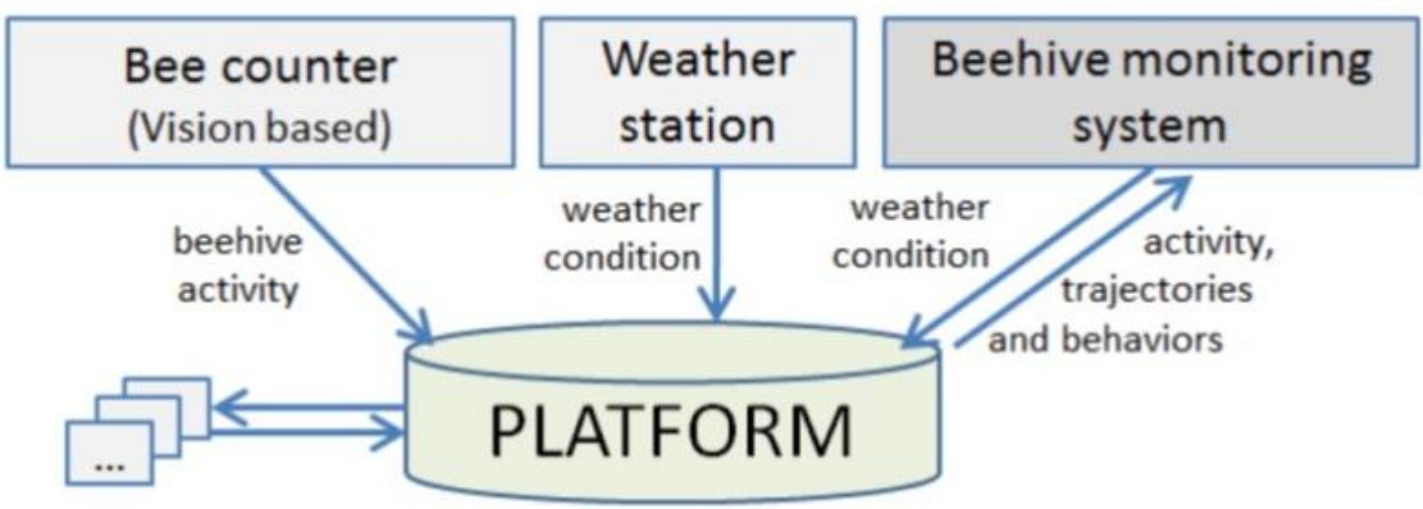

Figure 6. Platform for gathering information from different blocks on bee activity, bee trajectories and weather. The platform also allows for sharing of information with the beehive monitoring system. Source: (Chiron et al., 2013).

\subsection{Bioacoustic monitoring of poultry using biosensors}

Livestock farming and production do not simply target economic goals, but food quality, safety, broiler production efficiency and sustainability (Berckmans, 2006). For these purposes, a growing need emerges in livestock farming, particularly in chicken farming, to monitor and assess the animals' health, activity and welfare in real time, efficiently and economically. 
878 Current, traditional monitoring systems in poultry farming are based on manual methods or

879 simple systems relying on the observation, judgment and experience of the farmers, which is

880 time-consuming, not real-time, and inaccurate.

881

882 Secondly, when it comes to the monitoring of the health, activity and welfare of chickens,

883 currently available methods and systems cannot meet the increasing technical, administrative, 884 and organisational requirements of ever-growing farms, which limits the possibility and 885 feasibility of monitoring their livestock (Halachmi and Guarino, 2016). Thirdly, when targeting 886 health and disease monitoring, clinical signs such as nasal discharge and diarrhoea are non887 specific and cannot be used as evidence in diagnosis (Rahimian et al., 2012); and advanced 888 diagnostic methods including ELISA and real-time RT-PCR are only useful when daily 889 monitoring shows the necessity, because of their requirements for expert personnel, expensive 890 equipment, and time-consuming and costly processes (Soltan et al., 2016).

891

892 Precision livestock farming and smart agriculture are calling for novel and automatic systems 893 that are effective, efficient and affordable, and which meet variable goals to satisfy the purposes 894 of monitoring the health, activity and welfare of chickens (Banakar et al., 2016). The ideal 895 system would be an automatic device with an alarm feature, which is technologically simplified 896 enough to be understandable and usable by farmers to assist in their daily monitoring of welfare, 897 such as activities and conditions of the birds

899 Recent development of scientific research and technology in chickens and other livestock 900 indicates that vocalisation monitoring could be a valuable tool for predicting diseases and 
901 enhancing productivity. Vocalisation technology is based on sounds made by the birds in their

902 daily activities on the farms. The acoustic monitoring system was first developed to monitor

903 coughing in porcine livestock farms, which found coughing to be an indicator of animal

904 conditions, as it is a frequent symptom of multiple respiratory diseases affecting the lungs of

905 livestock (Chedad et al., 2001). The new acoustic technology using neural networks as

906 classification methods can distinguish cough sounds from other sounds such as metal clanging,

907 grunts, and background noises. Using the nanobiosensors, vocalisation detection can be designed

908 and developed as an efficient, effective and affordable system in monitoring the health

909 conditions of chickens, to provide opportunities for making decisions and undertaking necessary

910 actions.

911

912 Further development of the acoustic technology to detect porcine coughing contributed to a user-

913 friendly computer software system called Cool Edit Program (Gutierrez et al., 2010). With the

914 PC software system installed in porcine livestock farms, the farmers can monitor and initially

915 diagnose pigs with wasting diseases on breeding farms in real time based on the online cough

916 counter algorithm. An efficient data-mining technology was used to detect and recognise sounds

917 from pigs with or without wasting diseases, and the sound data were collected in audio

918 surveillance systems (Chung et al., 2013). Post sound acquisition, the widely used Mel

919 Frequency Cepstrum Coefficient (MFCC) sound analysis technology was used to extract and

920 differentiate sound data in the data pre-processing phase. Further development of the coughing

921 sound recognition system used the Support Vector Data Description (SVDD) technology to

922 enhance the automatic monitoring system. The SVDD worked as an anomaly or novelty detector

923 to detect sound data related to porcine wasting diseases. Finally, the Sparse Representation 
924 Classifier (SRC) technology was used to classify the sound data based on their relations to

925 different sub-types of porcine wasting diseases. Using the combination of MFCC, SVDD and

926 SRC technology, the study yielded up to $94 \%$ disease detection and $91 \%$ classification accuracy

927 of porcine wasting diseases.

929 The vocalisation- and acoustic-detection technology developed for pig coughing systems cannot

930 be directly applied to poultry farms due to the types and the frequencies of the sounds the

931 chickens make. The early exploration of the relation between vocalisation and poultry livestock

932 welfare can be traced back to 1953 (Collias and Joos, 1953), and a great number of different

933 vocalisation sounds, up to 30, have been described for juvenile and adult chickens (Manteuffel et

934 al., 2004). The vocalisation system can allow the detection of signs of welfare as gakel-calls,

935 alarm calls, distress calls, and stress calls through their sound analysis, indicating the levels of

936 energy, frequency, and call duration, to detect normal welfare or impaired welfare. The socially

937 relevant utterances will depend on complex contexts, but not solely on reactions to simple

938 internal states and external stimuli. For example, the hens' calls can be differentiated from cocks'

939 calls, and food calls from brooding hens to their chicks have a higher probability of occurrence

940 when the chicks are not feeding or are at a distance (Wauters and Richard- Yris, 2002).

942 Bioacoustics from poultry farms can also help to identify genetic strains and sexes of the birds,

943 in which the genetic strains can be identified using the second formant frequency and the pitch of

944 the sounds; and the sexes can be identified using the second formant frequency apart from the

945 sounds (Pereira et al., 2015a). An in-depth and comprehensive development of the acoustic

946 system can detect the key vocalisation frequency and pattern changes in routine activities of the 
947 birds, and identify the age and weight of young broiler chickens based on the audio monitoring

948 and comparison of anticipated sound patterns (Fontana et al., 2016). Figure 7 shows a schematic

949 representation of how a well-designed intelligent technology using a vocalisation-detection

950 system can monitor welfare and diagnose diverse infectious diseases in farmed chickens. Sound

951 signals collected using microphones and a data collection card analysed by a neural network

952 pattern-recognition system can detect and diagnose necrotic enteritis derived from the infection

953 of Clostridium perfringens type A (Sadeghi et al., 2015). The diagnostic accuracy was $66.6 \%$ on

954 day 2 , and $100 \%$ on day 8 post the disease onset. Another study compared three different sound-

955 detecting systems in the diagnosis of three avian infectious diseases with heavy economic losses:

956 Newcastle Disease, Bronchitis Virus, and Avian Influenza (Banakar et al., 2016). The three

957 systems were sound and time frequency detection alone, the Support Vector Data Description

958 system (SVDD) (Chung et al., 2013), and an artificially intelligent device using SVDD as an

959 input for intelligent analysis (Sadeghi et al., 2015). The results showed that the diagnostic

960 accuracy from frequency detection was 41.4\%, from SVDD was $83.3 \%$, and from the artificially

961 intelligent device was $91.2 \%$. These results indicate that the combination of SVDD technology

962 and artificially intelligent technology could yield the most accurate diagnosis in detecting poultry

963 diseases from bioacoustics.

964

965

966

967

968
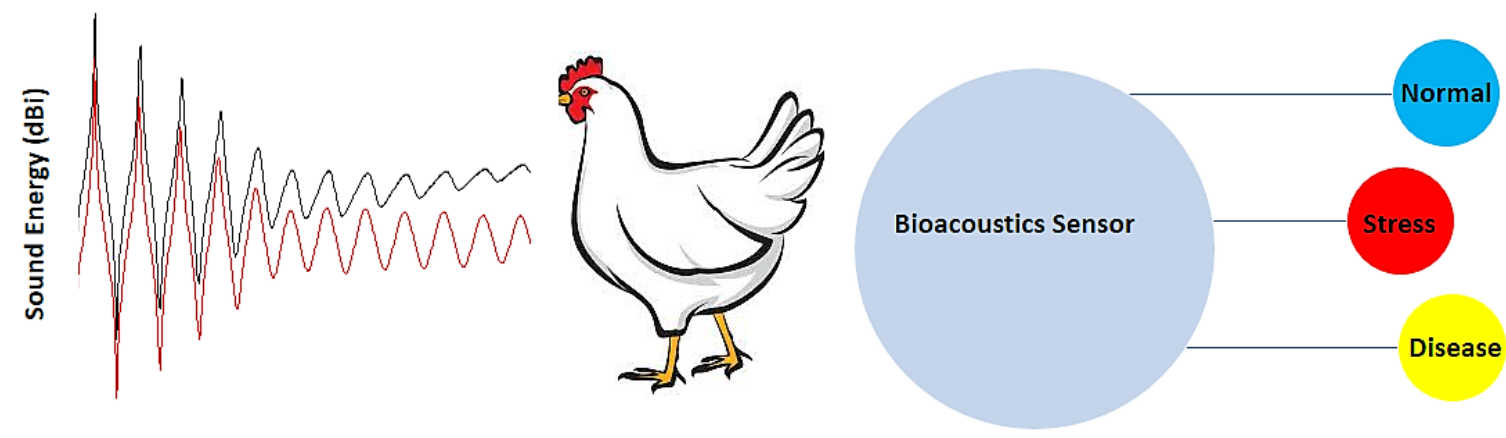

969

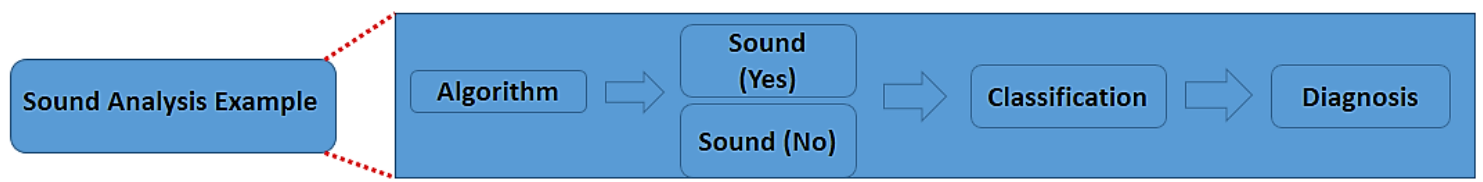


972 Figure 7. Schematic representation of the use of bioacoustics analysis for detection of wellbeing

973 in poultry livestock.

974

975 10. Perspectives

976

977 Precision livestock farming aims at creating a management system that relies upon autonomous,

978 continuous, real-time monitoring and control of all aspects of livestock management, including

979 reproduction, animal health and welfare, and the environmental impact of livestock production.

980 It is assumed that the direct monitoring of animals will achieve greater control over their health

981 status, which will eventually translate into better animal product quality over longer periods of

982 time. Biosensor technology shall enable accurate and affordable acquisition of data points, while

983 the smart algorithms, coupled with networked farms, shall further decision making and

984 management processes in the animal farms. The primary goal of precision livestock farming is to

985 generate reliable data using biosensors and run it through intelligent software systems to create

986 value for the farmer, the environment, and the animals in the form of improved animal health and

987 welfare, increased productivity and yields and reduced costs while minimising the impact on the

988 environment.

990 While the biosensor technology is available for individual parameters, key advancements in the 991 field are expected to generate robust monitoring systems for a multitude of parameters. Another

992 key challenge currently faced is the slow uptake of these technologies on commercial farms. This

993 has been attributed to the fact that although the precision systems and biosensors generate 
994 abundant data, the data is currently not being converted into useful information that could be

995 utilised for the decision-making process in livestock management. Furthermore, the economic

996 benefits of using these advanced systems is set to be demonstrated to individual farmers, who are

997 reluctant to make investments in these systems in the absence of a clear economic benefit.

999 There is no doubt that advancements in the development of nanobiosensors, combining 1000 nanotechnology with highly specific analytic techniques for metabolic biomolecules and

1001 surveillance systems for monitoring animal health and welfare will be ubiquitously used to 1002 manage livestock farms and prevent disease outbreak. The key challenges that remain to be 1003 resolved include harmonisation of methods across various platforms and large-scale 1004 implementation of data analysis and sharing technologies.

\begin{tabular}{lll}
\hline \multicolumn{1}{c}{ Biosensor } & Application & \multicolumn{1}{c}{ Reference } \\
\hline Grazing/Feeding & Pressure & (Braun et al., 2013; Nydegger et al., \\
& sensing & 2010; Pahl et al., 2016; Rutter et al., \\
& Acoustic & (Benvenutti et al., 2016; Navon et al., \\
& sensing & 2013) \\
& Acceleration & (Giovanetti et al., 2017; Herinaina et \\
& sensors & al., 2016; Mattachini et al., 2016;
\end{tabular}


2013; Umemura et al., 2009)

\section{Breath Analysis}

Cortisol

Alpha amylase

Glucose sensing
Animal stress (Kim et al., 2011; Yamaguchi et al., (cattle) 2013) Reviewed in (Singh et al., 2014)

Animal stress (Fuentes et al., 2011)

(Pigs)

Pigs (Park et al., 2009)

Fish stress (Hibi et al., 2012; Wu et al., 2015;

Yonemori et al., 2009)

\section{Diseases}

Bovine Cattle (Heinze et al., 2009; Luo et al., 2010;

Diarrhoea $\quad$ Montrose et al., 2015)

Virus

Avian Poultry (Bai et al., 2012; Diouani et al., Influenza Virus 2008; Guo et al., 2013; Li et al., 2011; Wang et al., 2009; Xu et al., 2007; Ye et al., 2014)

Foot-and- Swine (Gajendragad et al., 2001; Gomes et

Mouth Disease

al., 1999; Niedbalski, 2016; Salomón

et al., 2014; Yang et al., 2015; Yang et al., 2013)

Bovine
(Schaefer et al., 2012; Tarasov et al., 
Respiratory

2016)

Disease

Uric Acid

Volatile Organic Mycobacterium

Compounds

bovis

Reproductive

Cattle oestrus

Health Monitoring

cycle

Integrative

Wireless

Monitoring

Beehive

Monitoring bee

Bees

health beehive

Physical stress
renal
metabolism

Cattle

(Ellis et al., 2014; Fend et al., 2005;

Peled et al., 2012)

(Andersson et al., 2016; Andersson et

al., 2015; Jónsson et al., 2011;

Vanrell et al., 2014; Vreeburg,

2010a; Zeidan et al., 2016)

Pigs (De Groot et al., 2012)

Management

environment

1009 Table 3. Biosensor Transducers and Applications

\begin{tabular}{llll|}
\hline Transducer system & Principle & Applications & \\
\hline SPR & Changes in refractive & Urea detection & (Frasconi et al., \\
& index & Progesterone detection & 2009; Mitchell et \\
\hline
\end{tabular}




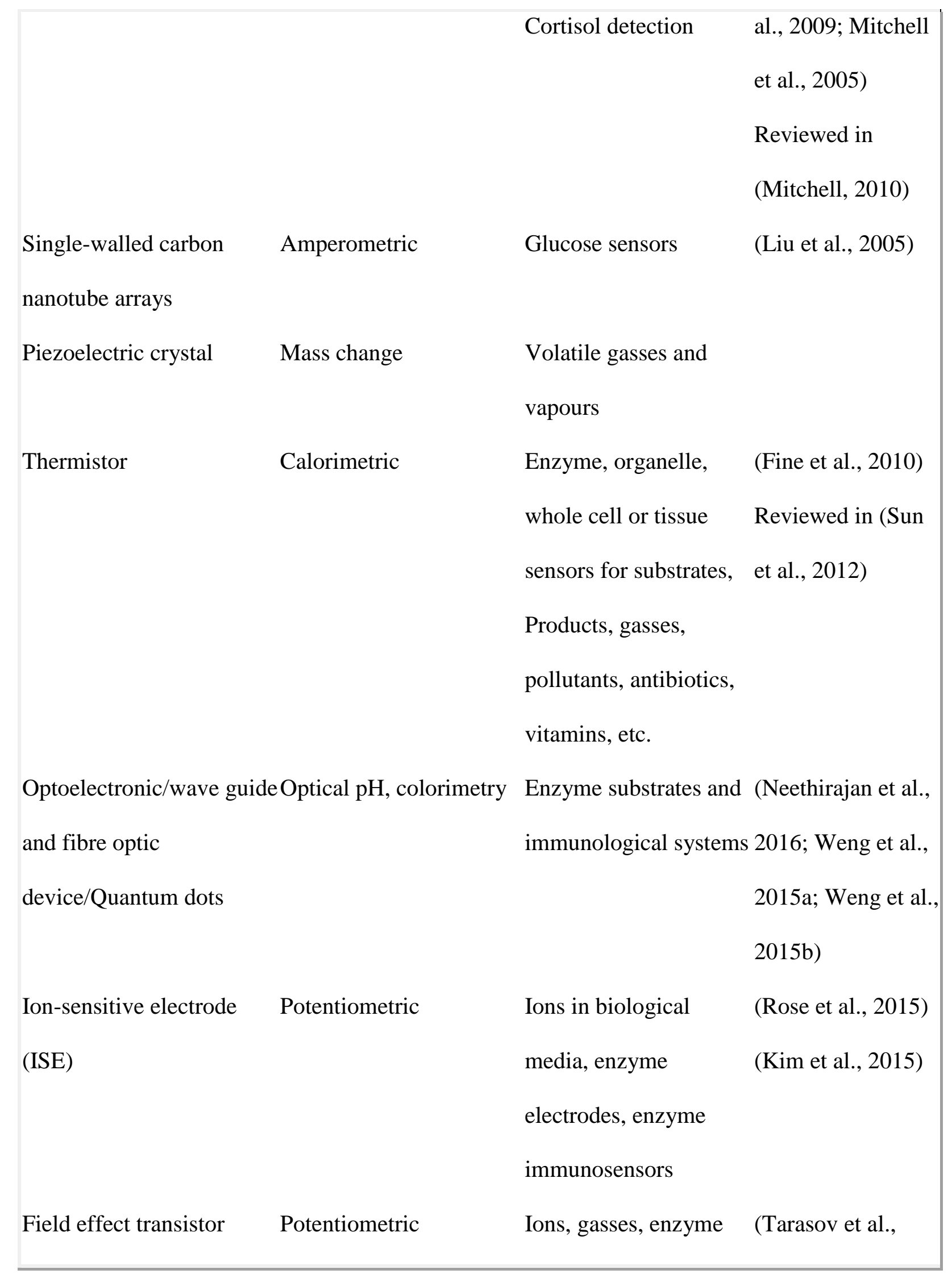




\section{Acknowledgments}

1015 The authors sincerely thank the Natural Sciences and Engineering Research Council of Canada 1016 (400929) and the Ontario Ministry of Agriculture, Food and Rural Affairs (300512) for funding 1017 this study.

Alexandratos, N., Bruinsma, J., 2012. World agriculture towards 2030/2050: the 2012 revision.

1023 Andersson, L.M., Okada, H., Miura, R., Zhang, Y., Yoshioka, K., Aso, H., Itoh, T., 2016. 1024 Wearable wireless estrus detection sensor for cows. Computers and Electronics in Agriculture $1025127,101-108$.

1026 Andersson, L.M., Okada, H., Zhang, Y., Itoh, T., Miura, R., Yoshioka, K., 2015. Wearable 1027 wireless sensor for estrus detection in cows by conductivity and temperature measurements. 1028 IEEE SENSORS, pp. 1-4.

1029 Andriamandroso, A., Lebeau, F., Bindelle, J., 2015. Changes in biting characteristics recorded 1030 using the inertial measurement unit of a smartphone reflect differences in sward attributes. In: 
1031 M., G., D., B. (Eds.), Proceedings of the 7th European conference on Precision Livestock

1032 Farming, Precision Livestock Farming, pp. 283-289, Milan, Italy.

1033 Bai, H., Wang, R., Hargis, B., Lu, H., Li, Y., 2012. A SPR Aptasensor for Detection of Avian

1034 Influenza Virus H5N1. Sensors 12(9), 12506-12518.

1035 Banakar, A., Sadeghi, M., Shushtari, A., 2016. An intelligent device for diagnosing avian

1036 diseases. Comput. Electron. Agric. 127, 744-753.

1037 Bandodkar, A.J., Wang, J., 2014. Non-invasive wearable electrochemical sensors: a review.

1038 Trends in Biotechnology 32(7), 363-371.

1039 Barth, F.G., Hrncir, M., Tautz, J., 2005. Vibratory and airborne-sound signals in bee 1040 communication (Hymenoptera). Insect Sounds and Communication: Physiology, Behaviour, 1041 Ecology, and Evolution, pp. 421-436. CRC Press.

1042 Benvenutti, M.A., Pavetti, D.R., Poppi, D.P., Gordon, I.J., Cangiano, C.A., 2016. Defoliation 1043 patterns and their implications for the management of vegetative tropical pastures to control 1044 intake and diet quality by cattle. Grass and Forage Science 71(3), 424-436.

1045 Berckmans, D., 2006. Automatic on-line monitoring of animals by precision livestock farming. 1046 Livestock production and society 287.

1047 Bhatta, D., Villalba, M.M., Johnson, C.L., Emmerson, G.D., Ferris, N.P., King, D.P., Lowe, 1048 C.R., 2012. Rapid Detection of Foot-and-Mouth Disease Virus with Optical Microchip Sensors. 1049 Procedia Chemistry 6, 2-10.

1050 Blom, J., Ridder, C., 2010. Reproductive Management and Performance Can be Improved by 1051 Use of DeLaval Herd Navigator®. The First North American Conference on Precision Dairy 1052 Management. 
1053 Braun, U., Trösch, L., Nydegger, F., Hässig, M., 2013. Evaluation of eating and rumination

1054 behaviour in cows using a noseband pressure sensor. BMC Veterinary Research 9(1), 164.

1055 Bromenshenk, J.J., Henderson, C.B., Seccomb, R.A., Welch, P.M., Debnam, S.E., Firth, D.R.,

1056 2015. Bees as biosensors: chemosensory ability, honey bee monitoring systems, and emergent

1057 sensor technologies derived from the pollinator syndrome. Biosensors 5(4), 678-711.

1058 Burciaga-Robles, L.O., Holland, B.P., Step, D.L., Krehbiel, C.R., McMillen, G.L., Richards, 1059 C.J., Sims, L.E., Jeffers, J.D., Namjou, K., McCann, P.J., 2009. Evaluation of breath biomarkers 1060 and serum haptoglobin concentration for diagnosis of bovine respiratory disease in heifers newly 1061 arrived at a feedlot. American journal of veterinary research 70(10), 1291-1298.

1062 Caja, G., Castro-Costa, A., Knight, C.H., 2016. Engineering to support wellbeing of dairy 1063 animals. Journal of Dairy Research 83(2), 136-147.

1064 Calvet, S., Campelo, J.C., Estellés, F., Perles, A., Mercado, R., Serrano, J.J., 2014. Suitability 1065 evaluation of multipoint simultaneous CO2 sampling wireless sensors for livestock buildings. 1066 Sensors 14(6), 10479-10496.

1067 Chedad, A., Moshou, D., Aerts, J.-M., Van Hirtum, A., Ramon, H., Berckmans, D., 2001. AP1068 animal production technology: recognition system for pig cough based on probabilistic neural 1069 networks. Journal of agricultural engineering research 79(4), 449-457.

1070 Chen, L., Ye, S., Cai, K., Zhang, C., Zhou, G., He, Z., Han, H., 2015a. An aqueous platinum 1071 nanotube based fluorescent immuno-assay for porcine reproductive and respiratory syndrome 1072 virus detection. Talanta 144, 324-328.

1073 Chen, Y., Arsenault, R., Napper, S., Griebel, P., 2015b. Models and Methods to Investigate 1074 Acute Stress Responses in Cattle. Animals 5(4), 0411. 
1075 Chen, Y., Huang, C.-H., Hou, C., Huo, D., Jin, G., 2013. Rapid and Label-Free Detection of 1076 Porcine Reproductive and Respiratory Syndrome Virus on Nanoscale by Biosensor Based on 1077 Imaging Ellipsometry. Integrated Ferroelectrics 145(1), 122-129.

1078 Chepete, H., Xin, H., Mendes, L., Li, H., Bailey, T., 2012. Ammonia emission and performance 1079 of laying hens as affected by different dosages of Yucca schidigera in the diet. The Journal of 1080 Applied Poultry Research 21(3), 522-530.

1081 Chiron, G., Gomez-Krämer, P., Ménard, M., 2013. Outdoor 3D Acquisition System for Small 1082 and Fast Targets. Application to honeybee monitoring at the beehive entrance. GEODIFF 2013, 1083 pp. 10-19, Barcelona, France.

1084 Christensen, L.S., Brehm, K.E., Skov, J., Harlow, K.W., Christensen, J., Haas, B., 2011. 1085 Detection of foot-and-mouth disease virus in the breath of infected cattle using a hand-held 1086 device to collect aerosols. Journal of virological methods 177(1), 44-48.

1087 Chung, Y., Oh, S., Lee, J., Park, D., Chang, H.-H., Kim, S., 2013. Automatic detection and 1088 recognition of pig wasting diseases using sound data in audio surveillance systems. Sensors 1089 13(10), 12929-12942.

1090 Clapham, W.M., Fedders, J.M., Beeman, K., Neel, J.P., 2011. Acoustic monitoring system to 1091 quantify ingestive behavior of free-grazing cattle. Computers and Electronics in Agriculture $109276(1), 96-104$.

1093 Collias, N., Joos, M., 1953. The spectrographic analysis of sound signals of the domestic fowl. 1094 Behaviour 5(1), 175-188.

1095 Cook, N.J., 2012. Review: Minimally invasive sampling media and the measurement of 1096 corticosteroids as biomarkers of stress in animals. Canadian Journal of Animal Science 92(3), $1097 \quad 227-259$. 
1098

1099

1100

1101

1102

1103

1104

1105

1106

1107

1108

1109

1110

1111

1112

1113

1114

1115

1116

1117

1118

1119

Da Silva, N., Zardoya, R., Santurde, G., Solana, A., Castro, J., 1995. Rapid and sensitive detection of the bovine viral diarrhea virus genome in semen. Journal of virological methods 55(2), 209-218.

Davis, J.D., 2007a. Remote characterization of locomotion, grazing and drinking behavior in beef cattle using GPS and ruminant temperature dynamics. ProQuest.

Davis, J.D.L., 2007b. Remote Characterization of Locomotion, Grazing and Drinking Behavior in Beef Cattle Using GPS and Ruminant Temperature Dynamics. Iowa State University.

De Groot, D., Slieker, R., Van Loo, P., Verheij, E., Lorentsen, H., Laursen, M., Markert, M., Masse, F., Bouwens, F., Integrative Wireless Monitoring of Minipigs. Newsletter 38 Autumn 2012 pp 11-17.

Dietlein, D.G., 1985. A Method for Remote Monitoring of Activity of Honeybee Colonies by Sound Analysis. Journal of Apicultural Research 24(3), 176-183.

Diouani, M.F., Helali, S., Hafaid, I., Hassen, W.M., Snoussi, M.A., Ghram, A., JaffrezicRenault, N., Abdelghani, A., 2008. Miniaturized biosensor for avian influenza virus detection. Materials Science and Engineering: C 28(5-6), 580-583.

Duarte, C., Costa, T., Carneiro, C., Soares, R., Jitariu, A., Cardoso, S., Piedade, M., Bexiga, R., Freitas, P., 2016. Semi-Quantitative Method for Streptococci Magnetic Detection in Raw Milk. Biosensors 6(2), 19.

Durkin, J., DeLaval, B.W., 2010. Heat detection: Trends and opportunities. Proc. Second North Am. Conf. Precision Dairy Management, Toronto, Canada, pp. 1-10.

Ellis, C.K., Stahl, R.S., Nol, P., Waters, W.R., Palmer, M.V., Rhyan, J.C., VerCauteren, K.C., McCollum, M., Salman, M.D., 2014. A pilot study exploring the use of breath analysis to 
1120 differentiate healthy cattle from cattle experimentally infected with Mycobacterium bovis. PLoS

1121 One 9(2), e89280.

1122 Evans, S.K., 2015. Electronic beehive monitoring-applications to research. Julius-Kühn-Archiv $11230(450), 121$.

1124 Fend, R., Geddes, R., Lesellier, S., Vordermeier, H.-M., Corner, L.A.L., Gormley, E., Costello,

1125 E., Hewinson, R.G., Marlin, D.J., Woodman, A.C., Chambers, M.A., 2005. Use of an Electronic 1126 Nose To Diagnose Mycobacterium bovis Infection in Badgers and Cattle. Journal of Clinical 1127 Microbiology 43(4), 1745-1751.

1128 Fine, G.F., Cavanagh, L.M., Afonja, A., Binions, R., 2010. Metal oxide semi-conductor gas 1129 sensors in environmental monitoring. Sensors 10(6), 5469-5502.

1130 Fontana, I., Tullo, E., Scrase, A., Butterworth, A., 2016. Vocalisation sound pattern 1131 identification in young broiler chickens. Animal : an international Journal of Animal Bioscience $113210(09), 1567-1574$.

1133 Force, A. T., 2015. Critical role of animal science research in food security and sustainability.

1134 The National Academies Press, Washington, DC.

1135 Frasconi, M., Mazzarino, M., Botre, F., Mazzei, F., 2009. Surface plasmon resonance 1136 immunosensor for cortisol and cortisone determination. Anal Bioanal Chem 394(8), 2151-2159.

1137 Frish, M.B., 2014. Current and emerging laser sensors for greenhouse gas sensing and leak 1138 detection. SPIE Sensing Technology+ Applications, pp. 91010H--91012. International Society 1139 for Optics and Photonics.

1140 Fuentes, M., Tecles, F., Gutiérrez, A., Otal, J., Martínez-Subiela, S., Cerón, J.J., 2011. Validation 1141 of an Automated Method for Salivary Alpha-Amylase Measurements in Pigs (Sus Scrofa 
1142 Domesticus) and its Application as a Stress Biomarker. Journal of Veterinary Diagnostic 1143 Investigation 23(2), 282-287.

1144 Fütő, P., Markus, G., Kiss, A., Adányi, N., 2012. Development of a Catalase-Based 1145 Amperometric Biosensor for the Determination of Increased Catalase Content in Milk Samples. 1146 Electroanalysis 24(1), 107-113.

1147 Gajendragad, M.R., Kamath, K.N.Y., Anil, P.Y., Prabhudas, K., Natarajan, C., 2001. 1148 Development and standardization of a piezo electric immunobiosensor for foot and mouth 1149 disease virus typing. Veterinary Microbiology 78(4), 319-330.

1150 Gao, W., Emaminejad, S., Nyein, H.Y.Y., Challa, S., Chen, K., Peck, A., Fahad, H.M., Ota, H., 1151 Shiraki, H., Kiriya, D., Lien, D.-H., Brooks, G.A., Davis, R.W., Javey, A., 2016. Fully integrated 1152 wearable sensor arrays for multiplexed in situ perspiration analysis. Nature 529(7587), 509-514.

1153 Garner, C.E., Smith, S., Bardhan, P.K., Ratcliffe, N.M., Probert, C.S., 2009. A pilot study of 1154 faecal volatile organic compounds in faeces from cholera patients in Bangladesh to determine 1155 their utility in disease diagnosis. Transactions of the Royal Society of Tropical Medicine and 1156 Hygiene 103(11), 1171-1173.

1157 Giovanetti, V., Decandia, M., Molle, G., Acciaro, M., Mameli, M., Cabiddu, A., Cossu, R., 1158 Serra, M.G., Manca, C., Rassu, S.P.G., Dimauro, C., 2017. Automatic classification system for 1159 grazing, ruminating and resting behaviour of dairy sheep using a tri-axial accelerometer. 1160 Livestock Science 196, 42-48.

1161 Gomes, P., Giralt, E., Andreu, D., 1999. Surface plasmon resonance screening of synthetic 1162 peptides mimicking the immunodominant region of $\mathrm{C}-\mathrm{S} 8 \mathrm{c} 1$ foot-and-mouth disease virus. 1163 Vaccine 18(3-4), 362-370. 
1164 Grant, S.A., Heits, B., Kleiboeker, S., 2006. Development of an Optical Biosensor Utilizing

1165 GoldNanoparticles to Detect Porcine Reproductive and Respiratory Syndrome Virus. Sensor

1166 Letters 4(3), 246-252.

1167 Gumus, A., Lee, S., Karlsson, K., Gabrielson, R., Winkler, D., Erickson, D., 2014. Real-time in 1168 vivo uric acid biosensor system for biophysical monitoring of birds. Analyst 139(4), 742-748.

1169 Guo, D., Zhuo, M., Zhang, X., Xu, C., Jiang, J., Gao, F., Wan, Q., Li, Q., Wang, T., 2013.

1170 Indium-tin-oxide thin film transistor biosensors for label-free detection of avian influenza virus

1171 H5N1. Analytica Chimica Acta 773, 83-88.

1172 Gutierrez, W., Kim, S., Kim, D., Yeon, S., Chang, H., 2010. Classification of porcine wasting

1173 diseases using sound analysis. Asian-Australasian Journal of Animal Sciences 23(8), 1096-1104.

1174 Halachmi, I., Guarino, M., 2016. Editorial: Precision livestock farming: a 'per animal'approach

1175 using advanced monitoring technologies. Animal : an international journal of animal bioscience

1176 10(9), 1482-1483.

1177 Hassouna, M., Robin, P., Charpiot, A., Edouard, N., Méda, B., 2013. Infrared photoacoustic

1178 spectroscopy in animal houses: Effect of non-compensated interferences on ammonia, nitrous

1179 oxide and methane air concentrations. Biosystems Engineering 114(3), 318-326.

1180 He, Y., Zhang, Y.-j., Xia, H., Geng, H., Ruan, J., Wang, M., 2009. Open-path online monitoring

1181 of ambient atmospheric co2 based on laser absorption spectrum. Spectroscopy and Spectral 1182 Analysis 29(1), 10-13.

1183 Hearps, A., Zhang, Z., Alexandersen, S., 2002. Evaluation of the portable Cepheid SmartCycler 1184 real-time PCR machine for the rapid diagnosis of foot-and-mouth disease. Veterinary Record $1185150(20), 625-628$. 

for rapid and sensitive detection of bovine viral diarrhea virus. Sensors and Actuators B:

1188 Chemical 138(2), 491-496.

1189 Hellsten, Y., Tullson, P.C., Richter, E.A., Bangsbo, J., 1997. Oxidation of urate in human 1190 skeletal muscle during exercise. Free radical biology \& medicine 22(1-2), 169-174.

1191 Herinaina, A.L., Bindelle, J., Mercatoris, B., Lebeau, F., 2016. A review on the use of sensors to 1192 monitor cattle jaw movements and behavior when grazing. Biotechnology, Agronomy, Society 1193 and Environment 23(S1), 273-286.

1194 Hibi, K., Hatanaka, K., Takase, M., Ren, H., Endo, H., 2012. Wireless biosensor system for real1195 time L-lactic acid monitoring in fish. Sensors 12(5), 6269-6281.

1196 Hodgkinson, J., Smith, R., Ho, W.O., Saffell, J.R., Tatam, R.P., 2013. Non-dispersive infra-red 1197 (NDIR) measurement of carbon dioxide at $4.2 \mu \mathrm{m}$ in a compact and optically efficient sensor. 1198 Sensors and Actuators B: Chemical 186, 580-588.

1199 Hogeveen, H., Kamphuis, C., Steeneveld, W., Mollenhorst, H., 2010. Sensors and Clinical 1200 Mastitis - The Quest for the Perfect Alert. Sensors (Basel, Switzerland) 10(9), 7991-8009.

1201 Holtkamp, D.J., Kliebenstein, J.B., Neumann, E.J., 2013. Assessment of the economic impact of 1202 porcine reproductive and respiratory syndrome virus on United States pork producers. JSHAP $120321(2), 72-84$.

1204 Iguchi, S., Kudo, H., Saito, T., Ogawa, M., Saito, H., Otsuka, K., Funakubo, A., Mitsubayashi, 1205 K., 2007. A flexible and wearable biosensor for tear glucose measurement. Biomedical 1206 Microdevices 9(4), 603-609.

1207 Ingram, B., Gavine, F., Lawson, P., 2005. Fish Health Management Guidelines for Farmed 1208 Murray Cod., Fisheries Victoria Research Report. 
1209 Jegadeesan, S., Venkatesan, G.P., 2016. Smart cow health monitoring, farm environmental 1210 monitoring and control system using wireless sensor networks. Int. J. Adv. Eng. Tech./Vol

1211 VII/Issue I/Jan-March 334, 339.

1212 Jia, W., Bandodkar, A.J., Valdés-Ramírez, G., Windmiller, J.R., Yang, Z., Ramírez, J., Chan, G., 1213 Wang, J., 2013. Electrochemical Tattoo Biosensors for Real-Time Noninvasive Lactate 1214 Monitoring in Human Perspiration. Analytical chemistry 85(14), 6553-6560.

1215 Jindal, K., Tomar, M., Gupta, V., 2012. CuO thin film based uric acid biosensor with enhanced 1216 response characteristics. Biosensors and Bioelectronics 38(1), 11-18.

1217 Jónsson, R., Blanke, M., Poulsen, N.K., Caponetti, F., Højsgaard, S., 2011. Oestrus detection in 1218 dairy cows from activity and lying data using on-line individual models. Computers and 1219 Electronics in Agriculture 76(1), 6-15.

1220 Kim, J., Imani, S., de Araujo, W.R., Warchall, J., Valdés-Ramírez, G., Paixão, T.R.L.C., 1221 Mercier, P.P., Wang, J., 2015. Wearable salivary uric acid mouthguard biosensor with integrated 1222 wireless electronics. Biosensors and Bioelectronics 74, 1061-1068.

1223 Kim, S.B., Takenaka, Y., Torimura, M., 2011. A Bioluminescent Probe for Salivary Cortisol. 1224 Bioconjugate Chemistry 22(9), 1835-1841.

1225 Knobloch, H., Köhler, H., Commander, N., Reinhold, P., Turner, C., Chambers, M., Pardo, M., 1226 Sberveglieri, G., 2009. Volatile organic compound (VOC) analysis for disease detection: proof 1227 of principle for field studies detecting paratuberculosis and brucellosis. AIP Conference 1228 Proceedings, pp. 195-197. AIP.

1229 Kridi, D.S., Carvalho, C.G.N.d., Gomes, D.G., 2016. Application of wireless sensor networks for 1230 beehive monitoring and in-hive thermal patterns detection. Comput. Electron. Agric. 127, 2211231 235. 
1232 Kumanan, V., Nugen, S.R., Baeumner, A.J., Chang, Y.-F., 2009. A biosensor assay for the 1233 detection of Mycobacterium avium subsp. paratuberculosis in fecal samples. J Vet Sci 10(1), 35123442.

1235 La Belle, J.T., Engelschall, E., Lan, K., Shah, P., Saez, N., Maxwell, S., Adamson, T., Abou-Eid, 1236 M., McAferty, K., Patel, D.R., Cook, C.B., 2014. A Disposable Tear Glucose Biosensor-Part 4: 1237 Preliminary Animal Model Study Assessing Efficacy, Safety, and Feasibility. J Diabetes Sci 1238 Technol 8(1), 109-116.

1239 Laca, WallisDeVries, 2000. Acoustic measurement of intake and grazing behaviour of cattle. 1240 Grass and Forage Science 55(2), 97-104.

1241 Lee, S.J., Kwon, Y.S., Lee, J.-e., Choi, E.-J., Lee, C.-H., Song, J.-Y., Gu, M.B., 2013. Detection 1242 of VR-2332 Strain of Porcine Reproductive and Respiratory Syndrome Virus Type II Using an 1243 Aptamer-Based Sandwich-Type Assay. Analytical chemistry 85(1), 66-74.

1244 Leonardi, S., Marchesi, G., Tangorra, F.M., Lazzari, M., 2013. Use of a proactive herd 1245 management system in a dairy farm of northern italy: technical and economic results. Journal of 1246 Agricultural Engineering 44(2s).

1247 Leopold, J.H., van Hooijdonk, R.T., Sterk, P.J., Abu-Hanna, A., Schultz, M.J., Bos, L.D., 2014. 1248 Glucose prediction by analysis of exhaled metabolites - a systematic review. BMC 1249 anesthesiology $14,46$.

1250 Li, D., Wang, J., Wang, R., Li, Y., Abi-Ghanem, D., Berghman, L., Hargis, B., Lu, H., 2011. A 1251 nanobeads amplified QCM immunosensor for the detection of avian influenza virus H5N1. 1252 Biosensors and Bioelectronics 26(10), 4146-4154. 
1253 Liu, J., Chou, A., Rahmat, W., Paddon- Row, M.N., Gooding, J.J., 2005. Achieving direct

1254 electrical connection to glucose oxidase using aligned single walled carbon nanotube arrays.

1255 Electroanalysis 17(1), 38-46.

1256 Lum, J., Wang, R., Lassiter, K., Srinivasan, B., Abi-Ghanem, D., Berghman, L., Hargis, B.,

1257 Tung, S., Lu, H., Li, Y., 2012. Rapid detection of avian influenza H5N1 virus using impedance

1258 measurement of immuno-reaction coupled with RBC amplification. Biosensors and

1259 Bioelectronics 38(1), 67-73.

1260 Luo, Y., Nartker, S., Miller, H., Hochhalter, D., Wiederoder, M., Wiederoder, S., Setterington,

1261 E., Drzal, L.T., Alocilja, E.C., 2010. Surface functionalization of electrospun nanofibers for

1262 detecting E. coli O157:H7 and BVDV cells in a direct-charge transfer biosensor. Biosensors and

1263 Bioelectronics 26(4), 1612-1617.

1264 Malon, R.S.P., Sadir, S., Balakrishnan, M., \#xf3, rcoles, E.P., 2014. Saliva-Based Biosensors:

1265 Noninvasive Monitoring Tool for Clinical Diagnostics. BioMed Research International 2014, 20.

1266 Manteuffel, G., Puppe, B., Schön, P.C., 2004. Vocalization of farm animals as a measure of

1267 welfare. Applied Animal Behaviour Science 88(1), 163-182.

1268 MarketsandMarkets, Precision Farming Market by Technology (Guidance System, Remote

1269 Sensing, Variable Rate Technology), Offering (Hardware Automation \& Control System, Sensor

1270 \& Monitoring Device, Software, Services), Application, and Geography - Global Forecast to

12712022

1272 Martinez-Pérez, D., Ferrer, M.L., Mateo, C.R., 2003. A reagent less fluorescent sol-gel

1273 biosensor for uric acid detection in biological fluids. Analytical Biochemistry 322(2), 238-242.

1274 Mattachini, G., Riva, E., Perazzolo, F., Naldi, E., Provolo, G., 2016. Monitoring feeding

1275 behaviour of dairy cows using accelerometers. Journal of Agricultural Engineering 47(1), 54-58. 
1276 Mazeris, F., 2010. DeLaval herd navigator: proactive herd management. Proceedings of First

1277 North American Conference on Precision Dairy Management, pp. 26-27.

1278 McNerney, R., Wondafrash, B.A., Amena, K., Tesfaye, A., McCash, E.M., Murray, N.J., 2010.

1279 Field test of a novel detection device for Mycobacterium tuberculosis antigen in cough. BMC

1280 infectious diseases $10,161$.

1281 Meikle, W., Holst, N., 2015. Application of continuous monitoring of honeybee colonies.

1282 Apidologie 46(1), 10-22.

1283 Mendes, L., Ogink, N., Edouard, N., van Dooren, H., Tinôco, I., Mosquera, J., 2015. NDIR Gas

1284 Sensor for Spatial Monitoring of Carbon Dioxide Concentrations in Naturally Ventilated

1285 Livestock Buildings. Sensors 15(5), 11239.

1286 Milone, D.H., Galli, J.R., Cangiano, C.A., Rufiner, H.L., Laca, E.A., 2012. Automatic

1287 recognition of ingestive sounds of cattle based on hidden Markov models. Computers and

1288 electronics in agriculture 87, 51-55.

1289 Mitchell, J., 2010. Small molecule immunosensing using surface plasmon resonance. Sensors

1290 (Basel, Switzerland) 10(8), 7323-7346.

1291 Mitchell, J.S., Lowe, T.E., Ingram, J.R., 2009. Rapid ultrasensitive measurement of salivary 1292 cortisol using nano-linker chemistry coupled with surface plasmon resonance detection. Analyst 1293 134(2), 380-386.

1294 Mitchell, J.S., Wu, Y., Cook, C.J., Main, L., 2005. Sensitivity enhancement of surface plasmon 1295 resonance biosensing of small molecules. Anal Biochem 343(1), 125-135.

1296 Montrose, A., Creedon, N., Sayers, R., Barry, S., O'riordan, A., 2015. Novel single gold 1297 nanowire-based electrochemical immunosensor for rapid detection of bovine viral diarrhoea 1298 antibodies in serum. Journal of Biosensors \& Bioelectronics 6(3), 1-7. 
1299 Mottram, T., Berry, P., Pickard, A., Hart, J.P., Pemberton, R., 2004. Non-invasive system for 1300 monitoring physiological or health status of animals by sampling saliva. Google Patents.

1301 Mottram, T., Dobbelaar, P., Schukken, Y., Hobbs, P., Bartlett, P., 1999. An experiment to 1302 determine the feasibility of automatically detecting hyperketonaemia in dairy cows. Livestock 1303 production science 61(1), 7-11.

1304 Nadin, L.B., Chopa, F.S., Gibb, M.J., Trindade, J.K.d., Amaral, G.A.d., de Faccio Carvalho, 1305 P.C., Gonda, H.L., 2012. Comparison of methods to quantify the number of bites in calves 1306 grazing winter oats with different sward heights. Applied Animal Behaviour Science 139(1-2), $1307 \quad 50-57$.

1308 Nakagawa, T., Hu, H., Zharikov, S., Tuttle, K.R., Short, R.A., Glushakova, O., Ouyang, X., Feig, 1309 D.I., Block, E.R., Herrera-Acosta, J., Patel, J.M., Johnson, R.J., 2006. A causal role for uric acid 1310 in fructose-induced metabolic syndrome. American journal of physiology. Renal physiology $1311290(3)$, F625-631.

1312 Navon, S., Mizrach, A., Hetzroni, A., Ungar, E.D., 2013. Automatic recognition of jaw 1313 movements in free-ranging cattle, goats and sheep, using acoustic monitoring. Biosystems 1314 Engineering 114(4), 474-483.

1315 Neethirajan, S., 2017. Recent advances in wearable sensors for animal health management. 1316 Sensing and Bio-Sensing Research 12, 15-29.

1317 Neethirajan, S., Weng, X., Chen, L., 2016. Biosensor for detection of subclinical ketosis. Google 1318 Patents.

1319 Neitzel, A.-C., Stamer, E., Junge, W., Thaller, G., 2014. Calibration of an automated California 1320 mastitis test with focus on the device-dependent variation. Springer Plus 3, 760. 
1321 Niedbalski, W., 2016. Recent progress in the diagnosis of foot-and-mouth disease: rapid field-

1322 based assays. Medycyna Weterynaryjna 72(6), 339-344.

1323 Nydegger, F., Gyga, L., Egli, W., 2010. Automatic measurement of rumination and feeding 1324 activity using a pressure sensor. p. 027. Cemagref, Aubiere.

1325 Nyhan, W.L., 1997. The recognition of Lesch-Nyhan syndrome as an inborn error of purine 1326 metabolism. Journal of inherited metabolic disease 20(2), 171-178.

1327 Ospina, P., Nydam, D., Stokol, T., Overton, T., 2010. Associations of elevated nonesterified fatty 1328 acids and $\beta$-hydroxybutyrate concentrations with early lactation reproductive performance and 1329 milk production in transition dairy cattle in the northeastern United States. Journal of dairy 1330 science 93(4), 1596-1603.

1331 Oudshoorn, F.W., Cornou, C., Hellwing, A.L.F., Hansen, H.H., Munksgaard, L., Lund, P., 1332 Kristensen, T., 2013. Estimation of grass intake on pasture for dairy cows using tightly and 1333 loosely mounted di- and tri-axial accelerometers combined with bite count. Comput. Electron. 1334 Agric. 99, 227-235.

1335 Pahl, C., Hartung, E., Grothmann, A., Mahlkow-Nerge, K., Haeussermann, A., 2016. Suitability 1336 of feeding and chewing time for estimation of feed intake in dairy cows. Animal : an 1337 international journal of animal bioscience 10(9), 1507-1512.

1338 Park, E.-J., Werner, J., Beebe, J., Chan, S., Barrie Smith, N., 2009. Noninvasive Ultrasonic 1339 Glucose Sensing with Large Pigs ( 200 Pounds) Using a Lightweight Cymbal Transducer Array 1340 and Biosensors. Journal of diabetes science and technology (Online) 3(3), 517-523.

1341 Park, M.-C., Ha, O.-K., 2015. Development of effective cattle health monitoring system based on 1342 biosensors. Advanced Science and Technology Letters 117, 180-185. 
1343 Peled, N., Ionescu, R., Nol, P., Barash, O., McCollum, M., VerCauteren, K., Koslow, M., Stahl,

1344 R., Rhyan, J., Haick, H., 2012. Detection of volatile organic compounds in cattle naturally

1345 infected with Mycobacterium bovis. Sensors and Actuators B: Chemical 171-172, 588-594.

1346 Pemberton, R.M., Hart, J.P., Mottram, T.T., 2001. An electrochemical immunosensor for milk

1347 progesterone using a continuous flow system1. Biosensors and Bioelectronics 16(9-12), 715-723.

1348 Pereira, E.M., Naeaes, I.D.A., Garcia, R.G., 2015a. Vocalization of broilers can be used to 1349 identify their sex and genetic strain. Engenharia Agrícola 35(2), 192-196.

1350 Pereira, J., Porto-Figueira, P., Cavaco, C., Taunk, K., Rapole, S., Dhakne, R., Nagarajaram, H., 1351 Camara, J.S., 2015b. Breath analysis as a potential and non-invasive frontier in disease 1352 diagnosis: an overview. Metabolites 5(1), 3-55.

1353 Persily, A.K., 2016. Field measurement of ventilation rates. Indoor Air 26(1), 97-111.

1354 Piccot, S.D., Masemore, S.S., Ringler, E.S., Srinivasan, S., Kirchgessner, D.A., Herget, W.F., 1355 1994. Validation of a method for estimating pollution emission rates from area sources using 1356 open-path FTIR spectroscopy and dispersion modeling techniques. Air \& Waste 44(3), 271-279.

1357 Pritchard, G., Kirkwood, G., Sayers, A., 2002. Detecting antibodies to infectious bovine 1358 rhinotracheitis and BVD virus infections using milk samples from individual cows. Veterinary 1359 record $150(6), 182-183$.

1360 Qandour, A., Ahmad, I., Habibi, D., Leppard, M., 2014. Remote beehive monitoring using 1361 acoustic signals.

1362 Rahimian, M., ZamaniI, M.A., Momtaz, H., Niazi, M., 2012. Detection and phylogenetic 1363 analysis of newcastle disease virus based on molecular techniques in broiler in Isfahan province.

1364 Research, P.M., 2014. Biosensor Market Will Reach US \$22,551.2 million in 2020. Persistence 1365 Market Research. 
Reusch, C.E., Kley, S., Casella, M., 2006. Home monitoring of the diabetic cat. Journal of Feline Medicine \& Surgery 8(2), 119-127.

1368 Rose, D.P., Ratterman, M.E., Griffin, D.K., Hou, L., Kelley-Loughnane, N., Naik, R.R., Hagen, 1369 J.A., Papautsky, I., Heikenfeld, J.C., 2015. Adhesive RFID Sensor Patch for Monitoring of 1370 Sweat Electrolytes. IEEE Transactions on Biomedical Engineering 62(6), 1457-1465.

1371 Ruiz-Garcia, L., Lunadei, L., Barreiro, P., Robla, I., 2009. A Review of Wireless Sensor 1372 Technologies and Applications in Agriculture and Food Industry: State of the Art and Current 1373 Trends. Sensors 9(6), 4728.

1374 Rutter, S., Champion, R., Penning, P., 1997. An automatic system to record foraging behaviour 1375 in free-ranging ruminants. Applied Animal Behaviour Science 54(2-3), 185-195.

1376 Rutter, S.M., 2000. Graze: A program to analyze recordings of the jaw movements of ruminants. 1377 Behavior Research Methods, Instruments, \& Computers 32(1), 86-92.

1378 Sa, J., Ju, M., Han, S., Kim, H., Chung, Y., Park, D., 2015. Detection of Low-Weight Pigs by 1379 Using a Top-View Camera. Proceedings of The fourth International Conference on Information 1380 Science and Cloud Computing (ISCC2015). 18-19 December 2015. Guangzhou, China. Online 1381 at http://pos. sissa. it/cgi-bin/reader/conf. cgi? confid= 264, id. 24.

1382 Sadeghi, M., Banakar, A., Khazaee, M., Soleimani, M., 2015. An Intelligent Procedure for the 1383 Detection and Classification of Chickens Infected by Clostridium Perfringens Based on their 1384 Vocalization. Revista Brasileira de Ciência Avícola 17(4), 537-544.

1385 Salomón, F., Tropea, S., Brengi, D., Hernández, A., Alamón, D., Parra, M., Longinotti, G., 1386 Ybarra, G., Lloret, P., Mass, M., Roberti, M., Lloret, M., Malatto, L., Moina, C., Fraigi, L., 1387 Melli, L., Cortina, M.E., Serantes, D.R., Ugalde, J.E., Ciocchini, A., Comerci, D.J., 2014. 
1388 Smartphone controlled platform for point-of-care diagnosis of infectious diseases. 2014 IEEE 9th

1389 IberoAmerican Congress on Sensors, pp. 1-4.

1390 Schaefer, A.L., Cook, N.J., Bench, C., Chabot, J.B., Colyn, J., Liu, T., Okine, E.K., Stewart, M.,

1391 Webster, J.R., 2012. The non-invasive and automated detection of bovine respiratory disease

1392 onset in receiver calves using infrared thermography. Research in Veterinary Science 93(2), 928-

1393935.

1394 Schazmann, B., Morris, D., Slater, C., Beirne, S., Fay, C., Reuveny, R., Moyna, N., Diamond,

1395 D., 2010. A wearable electrochemical sensor for the real-time measurement of sweat sodium

1396 concentration. Analytical Methods 2(4), 342-348.

1397 Sethi, S., Nanda, R., Chakraborty, T., 2013. Clinical Application of Volatile Organic Compound

1398 Analysis for Detecting Infectious Diseases. Clinical microbiology reviews 26(3), 462-475.

1399 Shao, K., Zhang, C., Ye, S., Cai, K., Wu, L., Wang, B., Zou, C., Lu, Z., Han, H., 2017. Near-

1400 infrared electrochemiluminesence biosensor for high sensitive detection of porcine reproductive

1401 and respiratory syndrome virus based on cyclodextrin-grafted porous Au/PtAu nanotube. Sensors

1402 and Actuators B: Chemical 240, 586-594.

1403 Singh, A., Kaushik, A., Kumar, R., Nair, M., Bhansali, S., 2014. Electrochemical sensing of 1404 cortisol: a recent update. Applied biochemistry and biotechnology 174(3), 1115-1126.

1405 Soltan, M.A., Tsai, Y.-L., Lee, P.-Y.A., Tsai, C.-F., Chang, H.-F.G., Wang, H.-T.T., Wilkes, 1406 R.P., 2016. Comparison of electron microscopy, ELISA, real time RT-PCR and insulated 1407 isothermal RT-PCR for the detection of Rotavirus group A (RVA) in feces of different animal 1408 species. Journal of virological methods 235, 99-104. 
1409 Soukup, M., Biesiada, I., Henderson, A., Idowu, B., Rodeback, D., Ridpath, L., Bridges, E.G.,

1410 Nazar, A.M., Bridges, K.G., 2012. Salivary uric acid as a noninvasive biomarker of metabolic

1411 syndrome. Diabetology \& metabolic syndrome 4(1), 14.

1412 Spinhirne, J.P., Koziel, J.A., Chirase, N.K., 2004. Sampling and analysis of volatile organic

1413 compounds in bovine breath by solid-phase microextraction and gas chromatography-mass

1414 spectrometry. Journal of chromatography. A 1025(1), 63-69.

1415 Stein, J.E., Greco, D.S., 2002. Portable blood glucose meters as a means of monitoring blood

1416 glucose concentrations in dogs and cats with diabetes mellitus. Clinical techniques in small

1417 animal practice 17(2), 70-72.

1418 Stringer, R.C., Schommer, S., Hoehn, D., Grant, S.A., 2008. Development of an optical

1419 biosensor using gold nanoparticles and quantum dots for the detection of Porcine Reproductive

1420 and Respiratory Syndrome Virus. Sensors and Actuators B: Chemical 134(2), 427-431.

1421 Su, X., Li, S.F.Y., Liu, W., Kwang, J., 2000. Piezoelectric quartz crystal based screening test for 1422 porcine reproductive and respiratory syndrome virus infection in pigs. Analyst 125(4), 725-730.

1423 Sun, Y.-F., Liu, S.-B., Meng, F.-L., Liu, J.-Y., Jin, Z., Kong, L.-T., Liu, J.-H., 2012. Metal oxide

1424 nanostructures and their gas sensing properties: a review. Sensors 12(3), 2610-2631.

1425 Tani, Y., Yokota, Y., Yayota, M., Ohtani, S., 2013. Automatic recognition and classification of 1426 cattle chewing activity by an acoustic monitoring method with a single-axis acceleration sensor.

1427 Computers and Electronics in Agriculture 92, 54-65.

1428 Tarasov, A., Gray, D.W., Tsai, M.Y., Shields, N., Montrose, A., Creedon, N., Lovera, P., 1429 O'Riordan, A., Mooney, M.H., Vogel, E.M., 2016. A potentiometric biosensor for rapid on-site 1430 disease diagnostics. Biosensors \& bioelectronics 79, 669-678. 
1431 Turner, C., Knobloch, H., Richards, J., Richards, P., Mottram, T.T.F., Marlin, D., Chambers,

1432 M.A., 2012. Development of a device for sampling cattle breath. Biosystems Engineering

$1433112(2), 75-81$.

1434 Umemura, K., Wanaka, T., Ueno, T., 2009. Technical note: Estimation of feed intake while

1435 grazing using a wireless system requiring no halter. J Dairy Sci 92(3), 996-1000.

1436 Ungar, E.D., Rutter, S.M., 2006. Classifying cattle jaw movements: comparing IGER behaviour

1437 recorder and acoustic techniques. Applied animal behaviour science 98(1), 11-27.

1438 Vanrell, S.R., Chelotti, J.O., Galli, J., Rufiner, H.L., Milone, D.H., 2014. 3d acceleration for heat

1439 detection in dairy cows. XLIII Jornadas Argentinas de Informática e Investigación Operativa

1440 (43JAIIO)-VI Congreso Argentino de AgroInformática (CAI)(Buenos Aires, 2014).

1441 Vreeburg, N., 2010a. Precision management on two Dutch dairy farms by use of Herd

1442 Navigator., First North American Conference on Precision Dairy Management, Toronto, Canada.

1443 Vreeburg, N., 2010b. Precision Management On Two Dutch Dairy Farms By Use Of Herd

1444 Navigator ${ }^{\circledR}$. First North American Conference on Precision Dairy Management, Toronto,

1445 Canada.

1446 Wang, R., Li, Y., 2013. Hydrogel based QCM aptasensor for detection of avian influenzavirus.

1447 Biosensors and Bioelectronics 42, 148-155.

1448 Wang, R., Wang, Y., Lassiter, K., Li, Y., Hargis, B., Tung, S., Berghman, L., Bottje, W., 2009.

1449 Interdigitated array microelectrode based impedance immunosensor for detection of avian

1450 influenza virus H5N1. Talanta 79(2), 159-164.

1451 Waters, R.A., Fowler, V.L., Armson, B., Nelson, N., Gloster, J., Paton, D.J., King, D.P., 2014.

1452 Preliminary validation of direct detection of foot-and-mouth disease virus within clinical samples 
1453 using reverse transcription loop-mediated isothermal amplification coupled with a simple lateral

1454 flow device for detection. PloS one 9(8), e105630.

1455 Wathes, C.M., Kristensen, H.H., Aerts, J.M., Berckmans, D., 2008. Is precision livestock

1456 farming an engineer's daydream or nightmare, an animal's friend or foe, and a farmer's panacea

1457 or pitfall? Computers and Electronics in Agriculture 64(1), 2-10.

1458 Wauters, A.-M., Richard- Yris, M.-A., 2002. Mutual influence of the maternal hen's food calling 1459 and feeding behavior on the behavior of her chicks. Developmental psychobiology 41(1), 25-36.

1460 Weng, X., Chen, L., Neethirajan, S., Duffield, T., 2015a. Development of quantum dots-based 1461 biosensor towards on-farm detection of subclinical ketosis. Biosensors and Bioelectronics 72, $1462 \quad 140-147$.

1463 Weng, X., Zhao, W., Neethirajan, S., Duffield, T., 2015b. Microfluidic biosensor for $\beta$ 1464 Hydroxybutyrate $(\beta \mathrm{HBA})$ determination of subclinical ketosis diagnosis. Journal of 1465 Nanobiotechnology 13(1), 13.

1466 Wheeler, E.F., Casey, K.D., Gates, R.S., Xin, H., Zajaczkowski, J.L., Topper, P.A., Liang, Y., 1467 Pescatore, A.J., 2006. Ammonia emissions from twelve US broiler chicken houses. Trans. 1468 ASABE 49(5), 1495-1512.

1469 Wilson, A., 2015. Advances in Electronic-Nose Technologies for the Detection of Volatile 1470 Biomarker Metabolites in the Human Breath. Metabolites 5(1), 140.

1471 Wu, H., Aoki, A., Arimoto, T., Nakano, T., Ohnuki, H., Murata, M., Ren, H., Endo, H., 2015.

1472 Fish stress become visible: A new attempt to use biosensor for real-time monitoring fish stress.

1473 Biosensors and Bioelectronics 67, 503-510.

1474 Wu, S., Zhu, Y., Cai, Q., Zeng, K., Grimes, C.A., 2007. A wireless magnetoelastic $\alpha$-amylase 1475 sensor. Sensors and Actuators B: Chemical 121(2), 476-481. 
$1476 \mathrm{Xu}$, J., Suarez, D., Gottfried, D.S., 2007. Detection of avian influenza virus using an

1477 interferometric biosensor. Analytical and Bioanalytical Chemistry 389(4), 1193-1199.

1478 Yamaguchi, M., Matsuda, Y., Sasaki, S., Sasaki, M., Kadoma, Y., Imai, Y., Niwa, D., Shetty, V.,

1479 2013. Immunosensor with fluid control mechanism for salivary cortisol analysis. Biosensors \&

1480 bioelectronics 41, 186-191.

1481 Yang, M., Caterer, N.R., Xu, W., Goolia, M., 2015. Development of a multiplex lateral flow

1482 strip test for foot-and-mouth disease virus detection using monoclonal antibodies. Journal of

1483 virological methods 221, 119-126.

1484 Yang, M., Goolia, M., Xu, W., Bittner, H., Clavijo, A., 2013. Development of a quick and simple

1485 detection methodology for foot-and-mouth disease virus serotypes O, A and Asia 1 using a

1486 generic RapidAssay Device. Virology Journal 10, 125-125.

1487 Yasuda, T., Yonemura, S., Tani, A., 2012. Comparison of the characteristics of small

1488 commercial NDIR CO2 sensor models and development of a portable CO2 measurement device.

1489 Sensors 12(3), 3641-3655.

1490 Ye, W.W., Tsang, M.-K., Liu, X., Yang, M., Hao, J., 2014. Upconversion Luminescence

1491 Resonance Energy Transfer (LRET)-Based Biosensor for Rapid and Ultrasensitive Detection of

1492 Avian Influenza Virus H7 Subtype. Small 10(12), 2390-2397.

1493 Yonemori, Y., Takahashi, E., Ren, H., Hayashi, T., Endo, H., 2009. Biosensor system for

1494 continuous glucose monitoring in fish. Analytica Chimica Acta 633(1), 90-96.

1495 Zeidan, E., Shivaji, R., Henrich, V.C., Sandros, M.G., 2016. Nano-SPRi Aptasensor for the 1496 Detection of Progesterone in Buffer. Scientific Reports 6, 26714.

1497 Zhao, Y., Pan, Y., Rutherford, J., Mitloehner, F.M., 2012. Estimation of the interference in 1498 multi-gas measurements using infrared photoacoustic analyzers. Atmosphere 3(2), 246-265. 


\section{Highlights}

- Advances in the signaling strategies and data acquisition for animal health management are summarized.

- Review focuses on the systems for observing physiological parameters and health of livestock.

- Nanomaterials based development and application of biosensors for animal health are discussed.

- Advancement in non-invasive biosensing techniques for the monitoring of livestock are reviewed. 


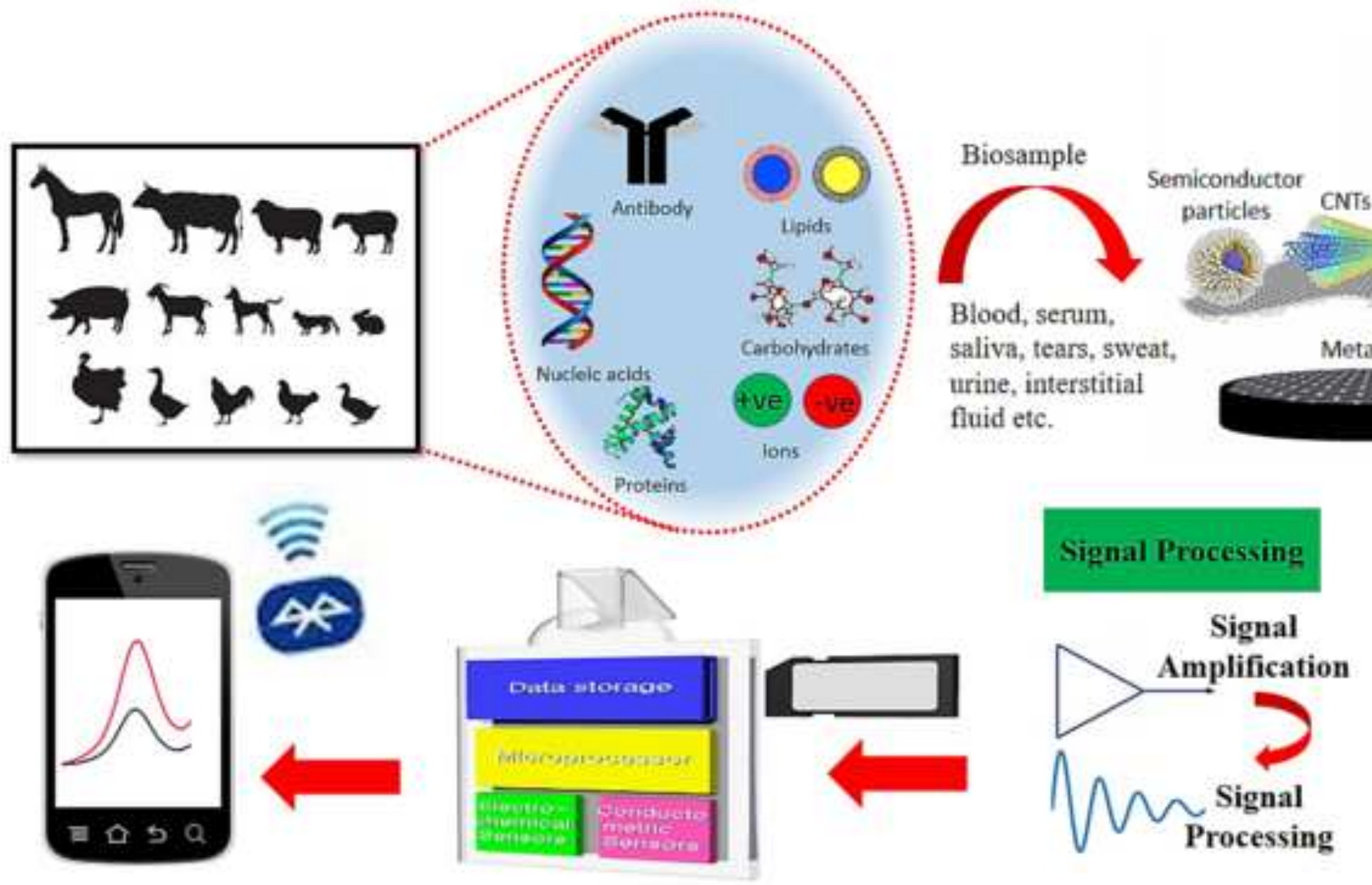

Transducer

Electrochemical, Optical, Colorimetric, Mass, SPR, Magnetic etc. 


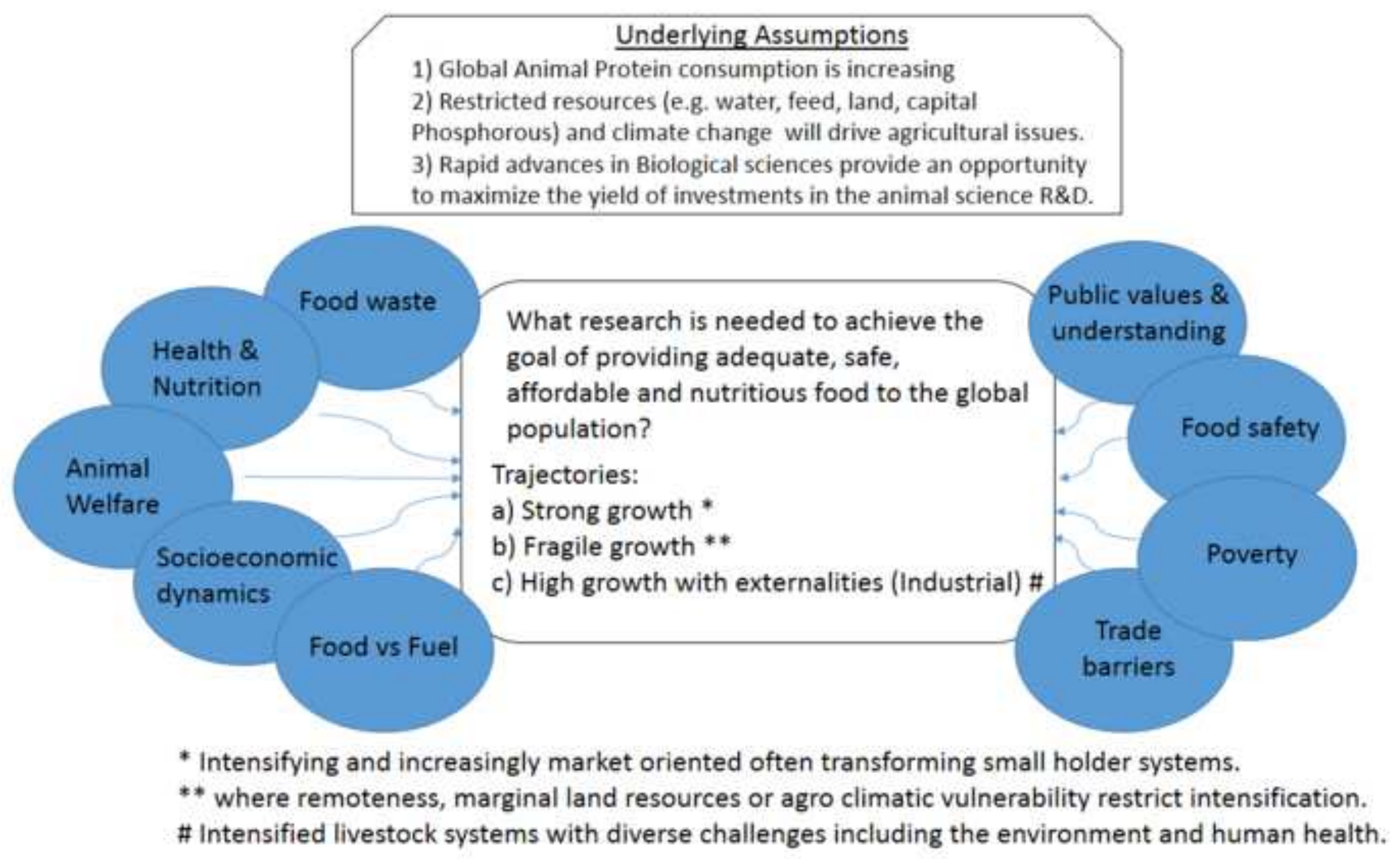




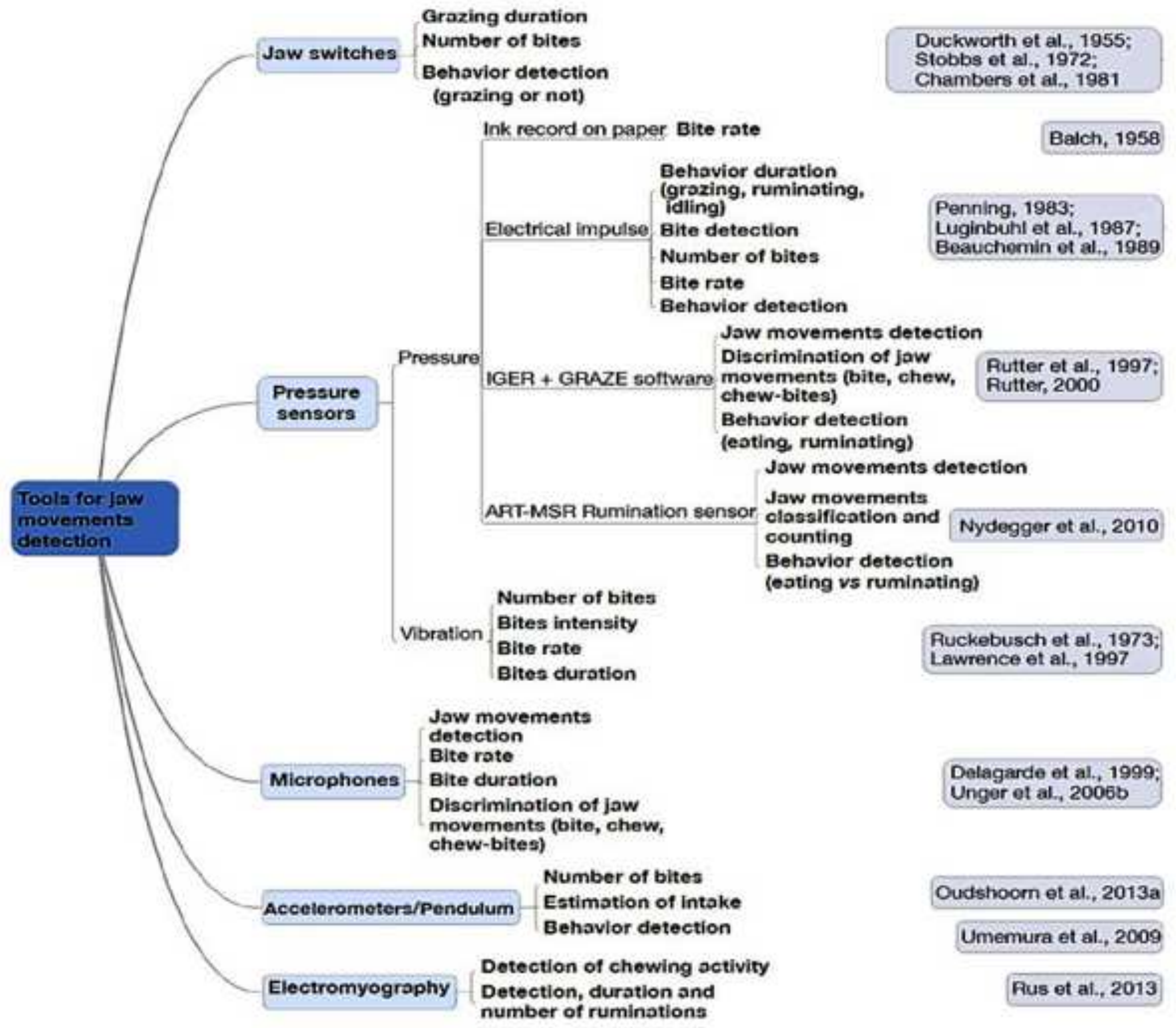

Behavior detection

novements fleation and

Behavior detection

Bites intensity

Bite rate

Jaw movements

Discrimination of jaw

novements (bite, chew.

Number of bites

udshoom et al., 2013a

Umemura et al., 2009

ber of ruminations 


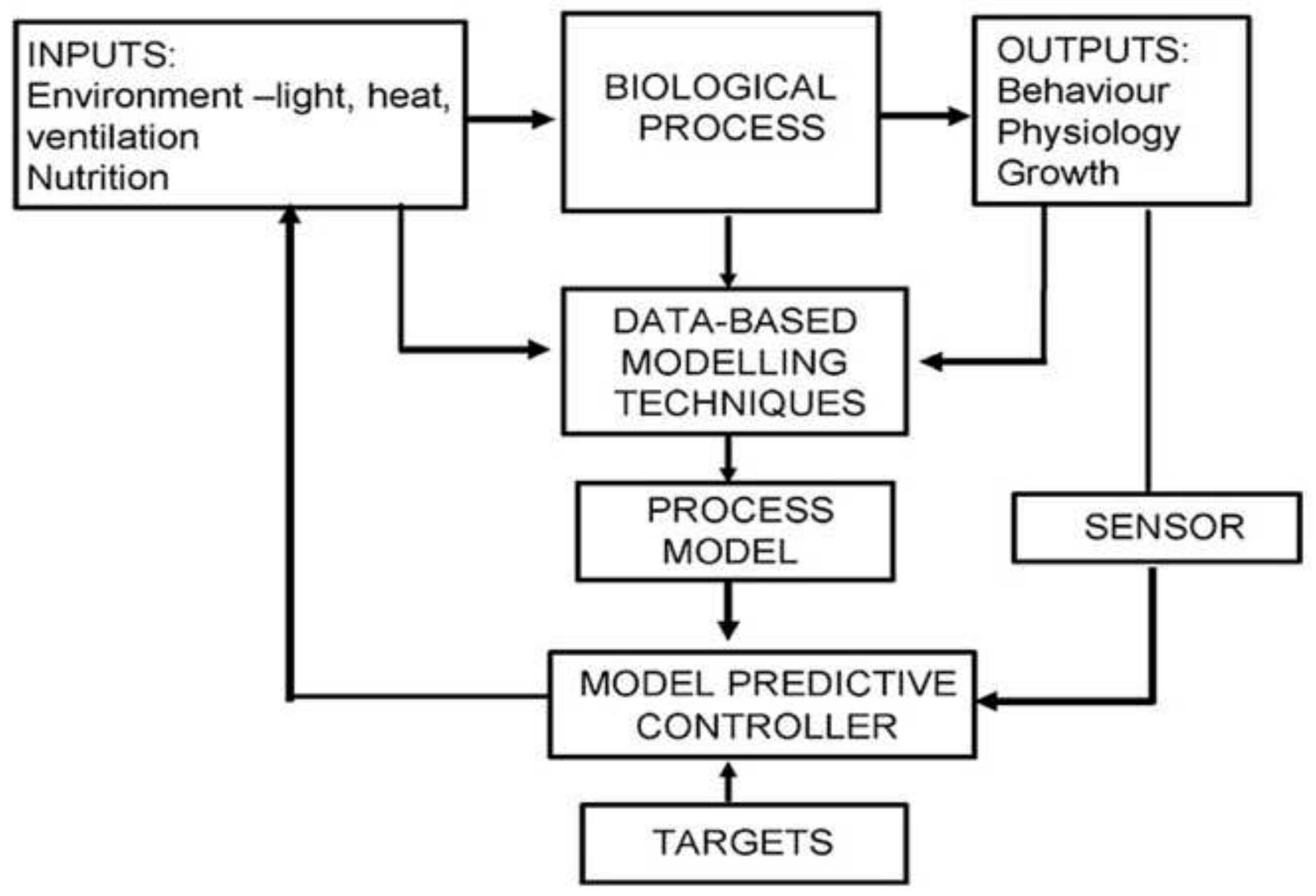




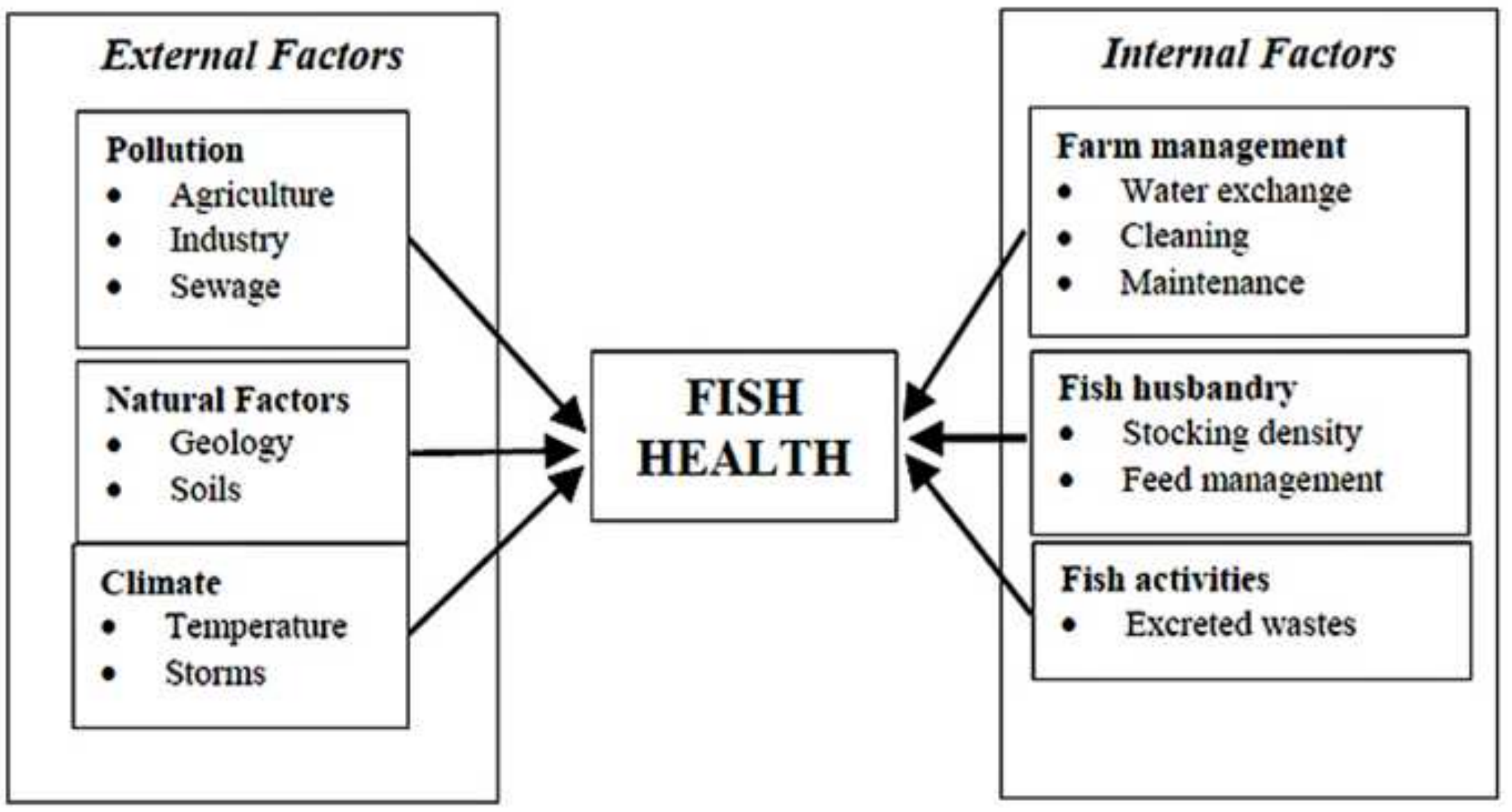




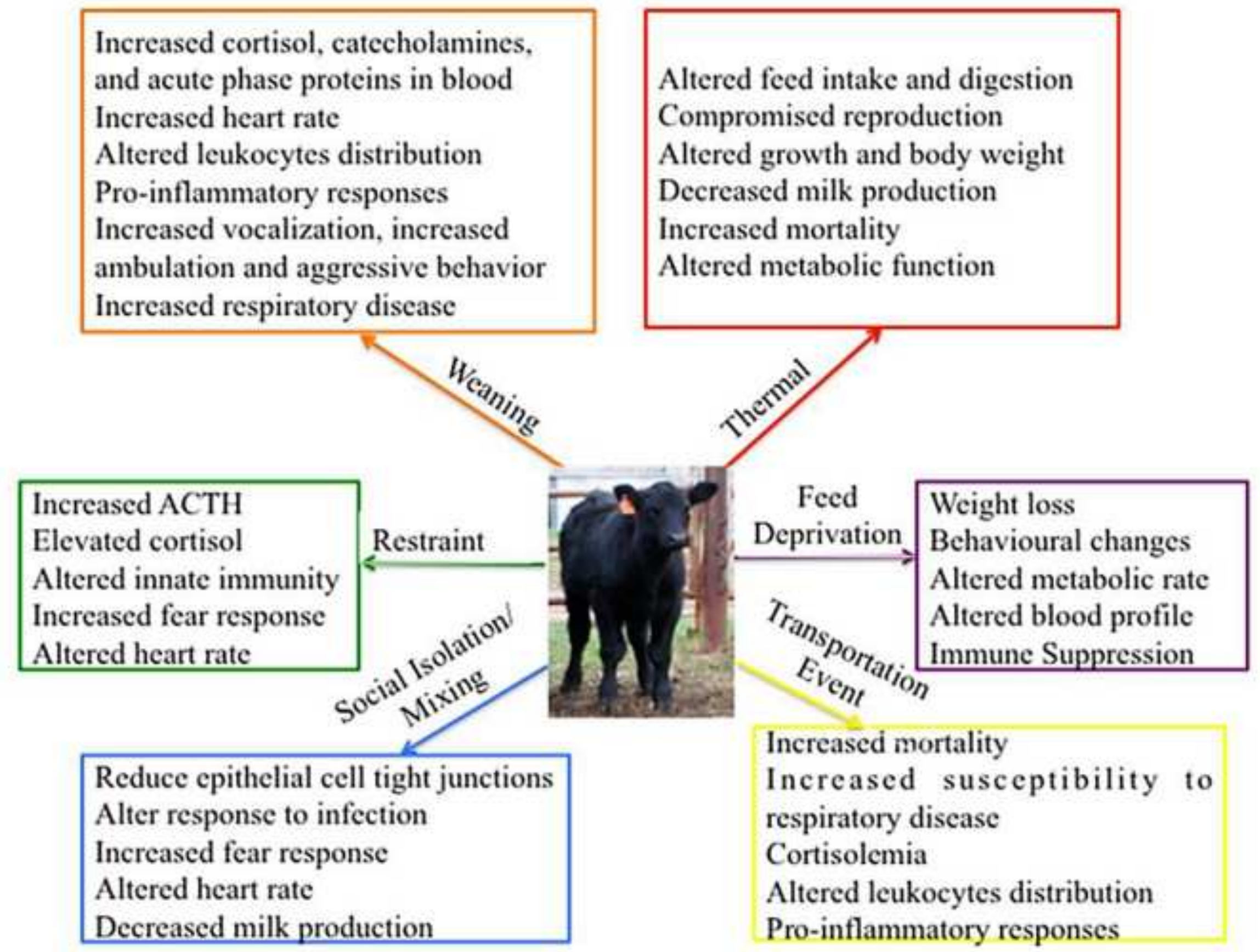




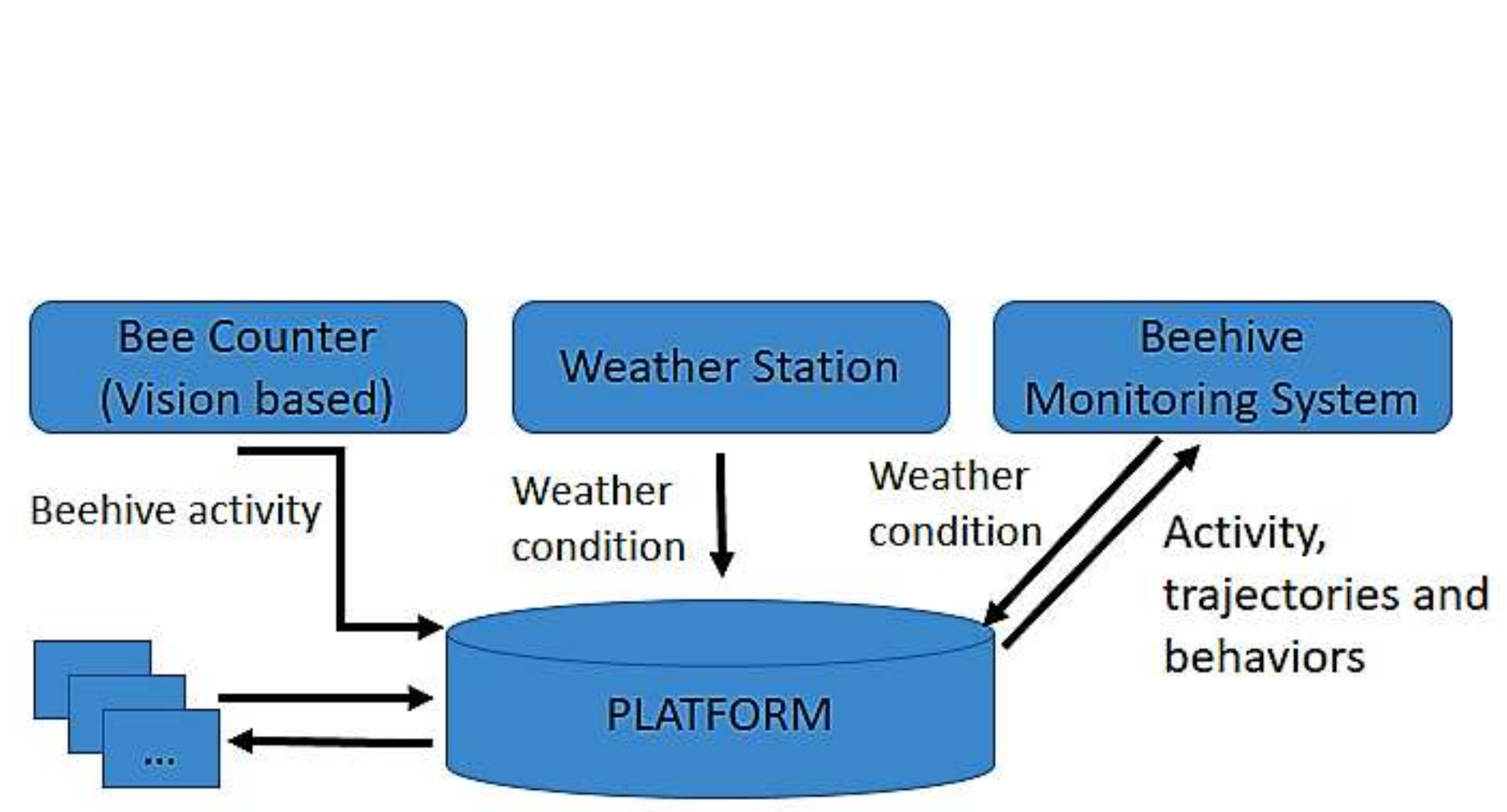


Click here to download high resolution image
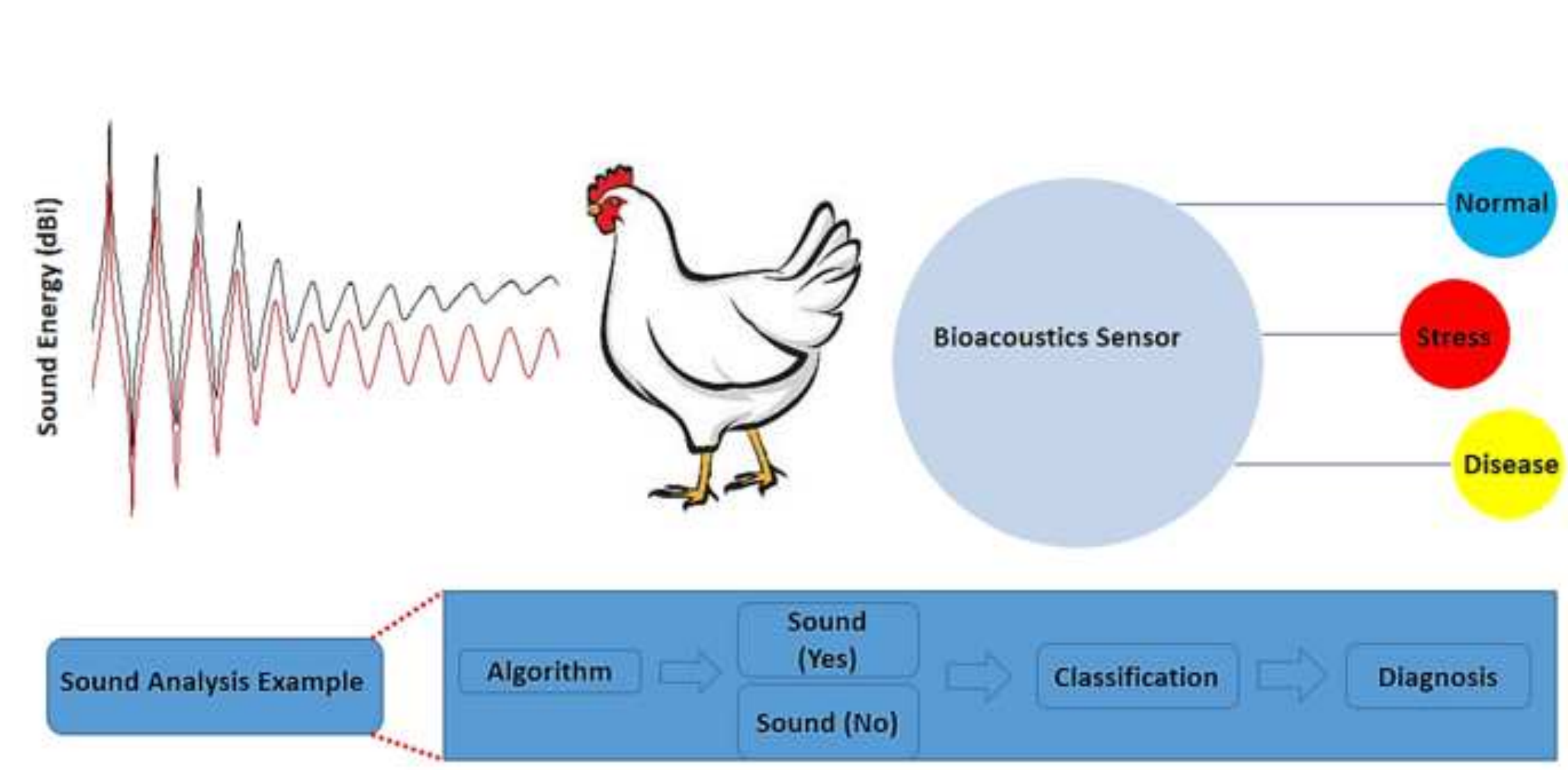